\title{
SENP7 senses oxidative stress to sustain metabolic fitness and antitumor functions of CD8 ${ }^{+} T$ cells
}

\author{
Zhongqiu Wu, ${ }^{1}$ Haiyan Huang, ${ }^{2}$ Qiaoqiao Han, ${ }^{1}$ Zhilin Hu, ${ }^{1}$ Xiao-Lu Teng, ${ }^{1}$ Rui Ding, ${ }^{1}$ Youqiong Ye, ${ }^{1}$ Xiaoyan Yu, ${ }^{1}$ Ren Zhao, ${ }^{2}$ \\ Zhengting Wang, ${ }^{3}$ and Qiang Zou'
}

'Shanghai Institute of Immunology, Department of Immunology and Microbiology, State Key Laboratory of Oncogenes and Related Genes, Hongqiao International Institute of Medicine, Tongren Hospital, Shanghai, China. '2Department of General Surgery, Ruijin Hospital, Shanghai, China. ${ }^{3}$ Department of Gastroenterology, Ruijin Hospital, Shanghai Jiao Tong University School of Medicine, Shanghai, China.

\begin{abstract}
The functional integrity of CD8 ${ }^{+} \mathrm{T}$ cells is tightly coupled to metabolic reprogramming, but how oxidative stress directs CD8 ${ }^{+}$ T cell metabolic fitness in the tumor microenvironment (TME) remains elusive. Here, we report that SUMO-specific protease 7 (SENP7) senses oxidative stress to maintain the CD8 ${ }^{+} \mathrm{T}$ cell metabolic state and antitumor functions. SENP7-deficient $\mathrm{CD8}^{+} \mathrm{T}$ cells exhibited decreased glycolysis and oxidative phosphorylation, resulting in attenuated proliferation in vitro and dampened antitumor functions in vivo. Mechanistically, CD8 ${ }^{+}$T cell-derived ROS triggered cytosolic SENP7-mediated PTEN deSUMOylation, thereby promoting PTEN degradation and preventing PTEN-dependent metabolic defects. Importantly, lowering T cell-intrinsic ROS restricted SENP7 cytosolic translocation and repressed CD8 ${ }^{+} \mathrm{T}$ cell metabolic and functional activity in human colorectal cancer samples. Our findings reveal that SENP7, as an oxidative stress sensor, sustains CD8+ $T$ cell metabolic fitness and effector functions and unveil an oxidative stress-sensing machinery in tumor-infiltrating CD8 ${ }^{+} \mathrm{T}$ cells.
\end{abstract}

\section{Introduction}

$\mathrm{CD}^{+} \mathrm{T}$ cells mediate potent antitumor functions and play a critical role in immunotherapy-induced immune responses against cancer (1-3). Functionality and metabolic fitness are central determinants of $\mathrm{CD}^{+} \mathrm{T}$ cell efficacy in cancer immunotherapy (4). Importantly, the functional integrity of $\mathrm{CD} 8^{+} \mathrm{T}$ cells is tightly coupled to metabolic reprogramming (5-7). Proliferating $\mathrm{CD}^{+} \mathrm{T}$ cells not only utilize aerobic glycolysis to support the rapid production of ATP but also engage mitochondrial metabolism to generate sufficient amounts of bioenergetic intermediates and fulfill bioenergetic and biosynthetic demands $(4,8,9)$. However, tumor-infiltrating $\mathrm{CD}^{+} \mathrm{T}$ cells typically undergo metabolic exhaustion, which is associated with $\mathrm{T}$ cell dysfunction, in the tumor microenvironment (TME) (10-13). The mechanism by which $\mathrm{CD}^{+} \mathrm{T}$ cell metabolic and functional activity is metabolically regulated in the TME remains unclear.

Oxidative stress, which is reflected by elevated levels of ROS in the TME, is a hallmark of many cancers $(14,15)$. Physiological levels of ROS are important for T cell activation, expansion, and effector function (16-19). Specific ROS-dependent signaling events are needed for $\mathrm{T}$ cell activation in vitro and antigen-specific $\mathrm{T}$ cell expansion in vivo (18). Persistent dysfunction of oxidative metabolism leads to impaired $\mathrm{T}$ cell antitumor functions in vivo (20). On the other hand, increased ROS production induced by mitochondrial dysfunction drives T cell exhaustion, and ROS neutralization slows T cell terminal differentiation (21). Notably, the mechanism by which oxidative stress shapes $\mathrm{CD} 8^{+} \mathrm{T}$ cell metabolic and functional fitness

Conflict of interest: The authors have declared that no conflict of interest exists. Copyright: () 2022, Wu et al. This is an open access article published under the terms of the Creative Commons Attribution 4.0 International License.

Submitted: September 21, 2021; Accepted: February 8, 2022; Published: April 1, 2022. Reference information: J Clin Invest. 2022;132(7):e155224.

https://doi.org/10.1172/JCl155224. in the TME remains elusive. Clarifying the oxidative stress-sensing machinery of tumor-infiltrating $\mathrm{CD}^{+} \mathrm{T}$ cells would be valuable.

SUMOylation is a reversible posttranslational modification (PTM) and regulates the subcellular localization, stability, and transcriptional activity of target proteins $(22,23)$. Notably, SUMOylation acts as a sensitive sensor of redox species and is strongly connected with oxidative stress (24). We and others have shown that SUMO-specific protease 3 (SENP3) is a redox sensor involved in multiple physiological and pathological processes $(15,25-27)$. Cytosolic accumulation of SENP3 is induced to enhance autophagosome formation in a ROS-dependent manner upon cellular starvation (28). Moreover, ROS-induced SENP3 protein stability is responsible for regulatory $\mathrm{T}$ cell maintenance and DC function in the TME $(15,27)$. SUMO-specific protease 7 (SENP7), another SUMO2/3-specific protease, is known for regulating epithelial-mesenchymal transition, DNA repair, and innate immune responses (29-32). However, whether SENP7 senses oxidative stress to affect the phenotype and function of tumor-infiltrating $\mathrm{CD}^{+} \mathrm{T}$ cells has not previously been defined.

Here, we present biochemical, genetic, and functional evidence that SENP7, as an oxidative stress sensor, sustains $\mathrm{CD}^{+} \mathrm{T}$ cell metabolic and functional states. We found that ROS-induced cytosolic translocation of SENP7 promoted PTEN degradation and prevented PTEN-dependent metabolic defects, thereby enhancing CD8 ${ }^{+} \mathrm{T}$ cell antitumor function. Accordingly, pharmacological targeting of ROS dampened the metabolic fitness of intratumoral $\mathrm{CD} 8^{+} \mathrm{T}$ cells and impaired their effector functions. These findings highlight the oxidative stress-sensing machinery of $\mathrm{CD} 8^{+} \mathrm{T}$ cells in the TME and suggest that targeting this process may improve cancer immunotherapy.

\section{Results}

ROS trigger cytosolic translocation of SENP7 in tumor-infiltrating $C D 8^{+} T$ cells. To investigate the expression profile of SENP7 in tumor-infiltrating $\mathrm{T}$ cells, we isolated $\mathrm{CD} 4^{+} \mathrm{T}$ cells and $\mathrm{CD} 8^{+} \mathrm{T}$ 
cells from patient-derived colorectal cancer (CRC) tissue for immunoblot analysis. Intriguingly, SENP7 was highly accumulated in tumor-infiltrating $\mathrm{CD} 8^{+} \mathrm{T}$ cells (Figure 1 , A and B). Similarly, SENP7 expression was greatly increased in $\mathrm{CD}^{+} \mathrm{T}$ cells from WT mice (Figure 1C). Notably, SENP7 cytosolic translocation was induced in $\mathrm{CD}^{+} \mathrm{T}$ cells upon activation by the TCR signals, although SENP7 protein levels were not altered (Figure 1D). Since TCR signals trigger ROS production in $\mathrm{CD}^{+} \mathrm{T}$ cells (33), we sought to determine the potential connection of ROS with SENP7 cytosolic translocation. Addition of $\mathrm{H}_{2} \mathrm{O}_{2}$ promoted the cytosolic translocation of SENP7 in $\mathrm{CD}^{+} \mathrm{T}$ cells (Figure 1E). Incubation of activated CD8 ${ }^{+}$ T cells with $\mathrm{N}$-acetylcysteine (NAC), a well-defined ROS inhibitor, prevented TCR-induced cytosolic translocation of SENP7 (Figure $1 F)$. Furthermore, tumor-infiltrating $\mathrm{CD}^{+} \mathrm{T}$ cells exhibited higher levels of ROS and cytosolic SENP7 than did splenic $\mathrm{CD}^{+} \mathrm{T}$ cells from tumor-bearing mice (Figure 1, G-I). In response to NAC treatment, SENP7 in tumor-infiltrating $\mathrm{CD}^{+} \mathrm{T}$ cells did not localize to the cytoplasm (Figure 1J). Importantly, the levels of ROS and cytosolic SENP7 in $\mathrm{CD}^{+} \mathrm{T}$ cells from human CRC tissue were higher than those in $\mathrm{CD}^{+} \mathrm{T}$ cells from PBMCs (Figure 1, K-M). Pharmacologic inhibition of ROS prevented SENP7 cytosolic translocation in $\mathrm{CD}^{+} \mathrm{T}$ cells from CRC tissue (Figure $1 \mathrm{~N}$ ). Collectively, these results indicate that ROS trigger SENP7 cytosolic translocation in tumor-infiltrating $\mathrm{CD}^{+} \mathrm{T}$ cells.

SENP7 ablation dampens $C D 8^{+} T$ cell antitumor responses in vivo. To explore the role of SENP7 in $\mathrm{CD}^{+} \mathrm{T}$ cell antitumor function, we crossed Senp $7^{f / f l}$ mice with Cd4-Cre mice to obtain Senp $7^{f / / f l}$ (designated WT) and Senp $7^{f / f l} C d 4$-Cre (designated KO) mice (Supplemental Figure 1A; supplemental material available online with this article; https://doi.org/10.1172/JCI155224DS1). The KO mice did not show obvious abnormalities in thymocyte development or peripheral $\mathrm{T}$ cell frequency (Supplemental Figure 1, B-D). In addition, the percentages of Tregs in the thymus, spleen, and lymph nodes were comparable between WT and KO mice (Supplemental Figure $1 \mathrm{E})$. Since $\mathrm{CD} 8^{+} \mathrm{T}$ cells play a crucial role in cell-mediated tumor killing (34), we inoculated WT and KO mice with MC38 murine colon cancer cells to determine whether SENP7 is required for antitumor $\mathrm{T}$ cell immune responses in vivo. Compared with WT mice, $\mathrm{KO}$ mice exhibited a profound increase in tumor size (Figure 2A). $\mathrm{CD}^{+} \mathrm{T}$ cells in the draining lymph nodes from tumor-bearing $\mathrm{KO}$ mice produced less IFN- $\gamma$ than did those from tumor-bearing WT mice, whereas the frequencies of IFN- $\gamma$-producing $\mathrm{CD}^{+}{ }^{+} \mathrm{T}$ cells in the draining lymph nodes were similar between tumor-bearing KO mice and tumor-bearing WT mice (Figure 2B). The tumor-bearing $\mathrm{KO}$ mice displayed decreased numbers of tumor-infiltrating $\mathrm{CD}^{+} \mathrm{T}$ cells (Figure $2 \mathrm{C}$ ). Moreover, the tumor-bearing $\mathrm{KO}$ mice had decreased frequencies of tumor-infiltrating IFN- $\gamma$-producing, TNF- $\alpha$-producing, and granzyme B-producing CD $8^{+} \mathrm{T}$ cells (Figure 2, D and E). By contrast, the frequencies of IFN- $\gamma$-producing $\mathrm{CD}^{+} \mathrm{T}$ cells in the tumors were similar between tumor-bearing $\mathrm{KO}$ mice and tumor-bearing WT mice (Supplemental Figure 2A). Parallel studies revealed that the KO mice also displayed impaired $\mathrm{CD}^{+} \mathrm{T}$ cell-mediated antitumor immunity in the B16-F10 murine melanoma model (Figure 2, F and G). To further confirm the CD8 ${ }^{+}$ $\mathrm{T}$ cell-specific role of SENP7 in antitumor function, we adoptively transferred OT-I cells isolated from Senp $7^{f / f l} C d 4$-Cre OT-I (KO OT-I) mice and their WT OT-I littermates into WT mice bearing chicken OVA-expressing MC38 (MC38-OVA) tumors. As expected, mice transferred with KO OT-I T cells displayed a weaker suppression of tumor growth and tumor-induced lethality (Figure 2, $\mathrm{H}$ and I). However, the tumor size and tumor-induced lethality were similar between the recipients of WT OT-II cells and KO OT-II cells (Supplemental Figure 2, B and C), indicating that SENP7 is dispensable for $\mathrm{CD} 4^{+} \mathrm{T}$ cell antitumor function. These results suggest a CD8 ${ }^{+} \mathrm{T}$ cell-specific role of SENP7 in antitumor function in vivo.

SENP7 is indispensable for $C D 8^{+} T$ cell proliferation in vivo and in vitro. To clarify the mechanism underlying the reduced antitumor activity of SENP7-deficient $\mathrm{CD}^{+} \mathrm{T}$ cells, we isolated tumorinfiltrating $\mathrm{CD}^{+} \mathrm{T}$ cells from tumor-bearing $\mathrm{WT}$ and $\mathrm{KO}$ mice for transcriptomic analysis. Cell proliferation-related genes, including Mki67, Cdk1, Plk1, and Ccnb1, were downregulated in tumor-infiltrating SENP7-deficient CD8 ${ }^{+} \mathrm{T}$ cells (Figure 3A). Consistent with this finding, the percentage of $\mathrm{Ki}-67^{+}$tumor-infiltrating $\mathrm{CD} 8^{+} \mathrm{T}$ cells was lower in tumor-bearing $\mathrm{KO}$ mice (Figure $3 \mathrm{~B}$ ). By contrast, tumor-bearing WT and $\mathrm{KO}$ mice had similar percentages of $\mathrm{Ki}-67^{+}$ tumor-infiltrating $\mathrm{CD}^{+}{ }^{+} \mathrm{T}$ cells (Figure 3C). Although anti-programmed cell death 1 (anti-PD-1) treatment efficiently inhibited tumor progression in tumor-bearing WT mice, this efficiency was largely suppressed in tumor-bearing KO mice (Figure 3D), indicating that SENP7-dependent $\mathrm{CD}^{+}{ }^{+} \mathrm{T}$ cell proliferation contributed to the antitumor immune response to checkpoint blockade.

To confirm that SENP7 is required for $\mathrm{CD}^{+} \mathrm{T}$ cell proliferation, we examined $\mathrm{CD} 8^{+} \mathrm{T}$ cell activity in vitro. Indeed, expression of the cell proliferation marker Ki-67 after TCR and CD28 stimulation was attenuated in SENP7-deficient $\mathrm{CD}^{+} \mathrm{T}$ cells (Figure 3E). Consistent with this finding, defective proliferation of SENP7-deficient $\mathrm{CD}^{+} \mathrm{T}$ cells was observed after TCR and CD28 stimulation (Figure $3 \mathrm{~F})$. On the other hand, neither the induction of $\mathrm{T}$ cell activation markers (Supplemental Figure 3, A and B) nor the frequency of apoptotic cells (Figure 3G) was affected in SENP7-deficient CD8 ${ }^{+}$ T cells. Furthermore, the expression of Ki-67 in SENP7-deficient $\mathrm{CD}^{+}{ }^{+} \mathrm{T}$ cells was similar to that in WT CD $4^{+} \mathrm{T}$ cells (Supplemental Figure $3 C$ ). Therefore, SENP7 is indispensable for $\mathrm{CD}^{+} \mathrm{T}$ cell proliferation in vivo and in vitro.

SENP7 promotes glycolysis and oxidative phosphorylation in $C D 8^{+}$ $T$ cells. To explore SENP7-dependent transcriptional programs, we analyzed the gene expression profiles of tumor-infiltrating $\mathrm{CD}^{+}$ $\mathrm{T}$ cells. Intriguingly, the phosphoinositide 3-kinase/mTOR (PI3K/ mTOR) signaling pathway-related gene expression profile was altered in tumor-infiltrating SENP7-deficient $\mathrm{CD}^{+} \mathrm{T}$ cells (Figure 4A). We further performed RNA-Seq using naive CD8 ${ }^{+} \mathrm{T}$ cells activated in vitro to confirm the gene expression profiles. As expected, the levels of genes involved in the PI3K/mTOR signaling pathway were modulated in SENP7-deficient CD8 ${ }^{+} \mathrm{T}$ cells (Figure 4B). Immunoblot and flow cytometric analyses revealed that activation of mTORC1 (assessed by measuring the phosphorylation of ribosomal protein S6) and mTORC2 (assessed by measuring the phosphorylation of AKT at Ser473) was impaired in SENP7-deficient $\mathrm{CD}^{+} \mathrm{T}$ cells stimulated with anti-CD3 and anti-CD28 antibodies (Figure 4, C and D). Because the PI3K/mTOR signaling pathway mediates metabolic reprogramming to sustain $\mathrm{T}$ cell proliferation and effector function (34), we next assessed $\mathrm{CD}^{+} \mathrm{T}$ cell metabolic fitness. Interestingly, SENP7-deficient $\mathrm{CD} 8^{+} \mathrm{T}$ cells had significantly lower baseline and maximum glycolytic rates than did WT $\mathrm{CD}^{+} \mathrm{T}$ 
A

B

C

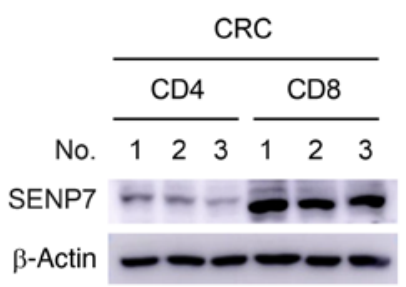

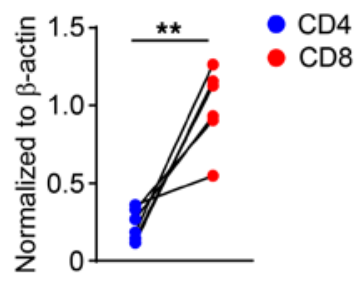

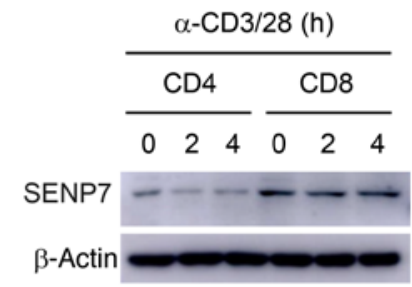

D

$$
\frac{\alpha-C D 3 / 28(h)}{0 \quad 0.5 \quad 1}
$$

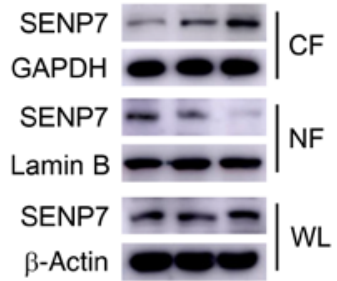

E

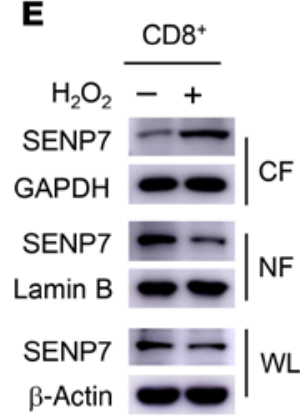

$\mathbf{F}$

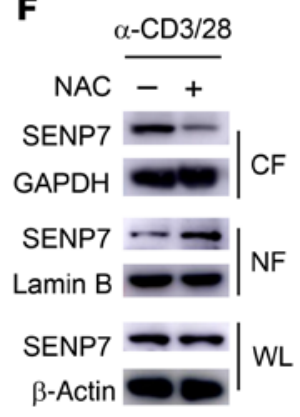

G
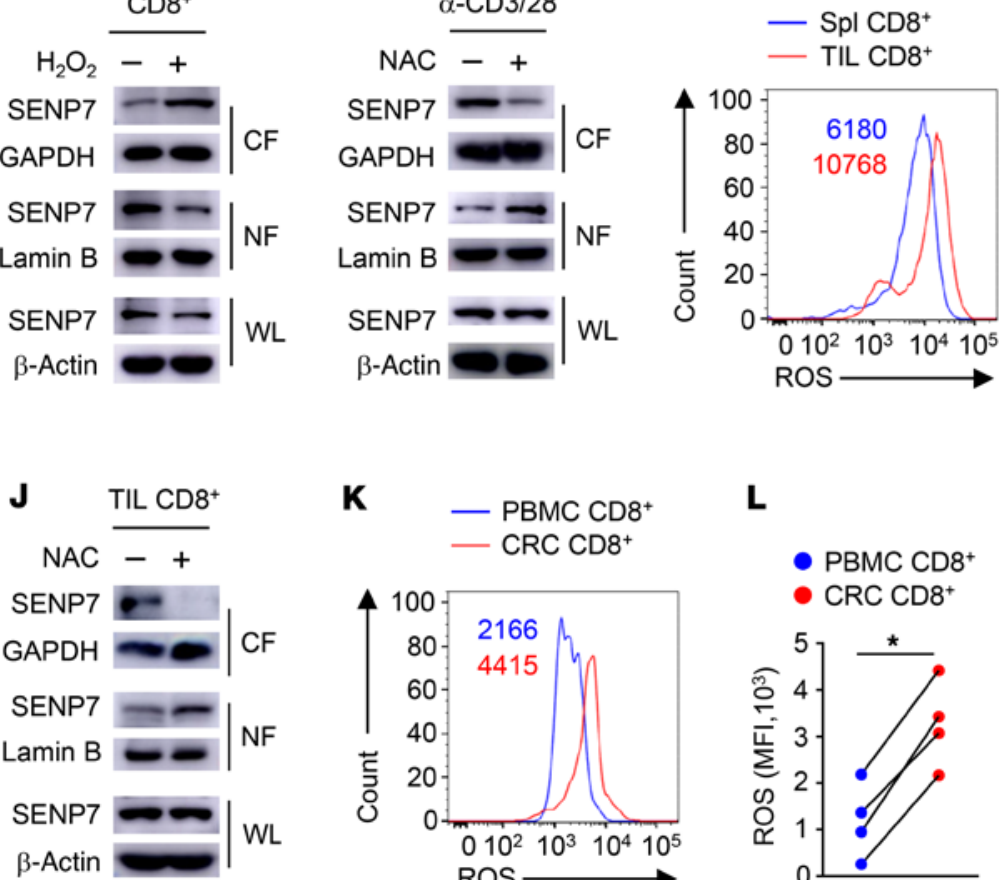

K
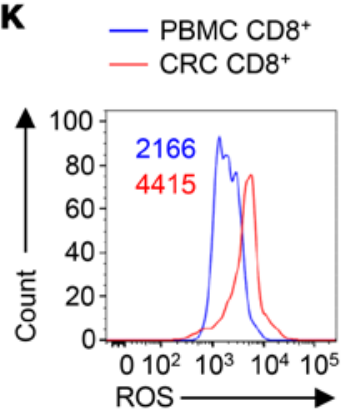

$\mathbf{L}$

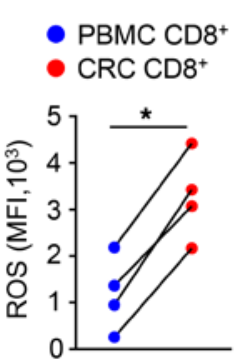

H

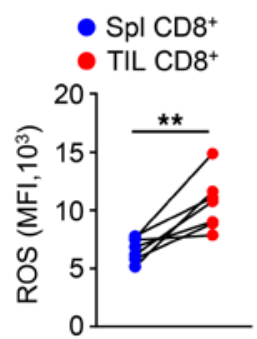

I

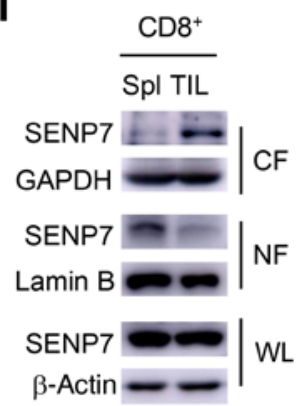

Figure 1. ROS trigger cytosolic translocation of SENP7 in tumor-infiltrating CD8+ $\mathbf{T}$ cells. (A and $\mathbf{B})$ Immunoblot analysis of the indicated proteins (A) and quantification of SENP7 expression $(\mathbf{B}, n=6)$ in $C D 4^{+}$and $C D 8^{+}$T cells from CRC tissues. (C) Immunoblot analysis of the indicated proteins in $C D 4^{+}$and CD8 ${ }^{+} \mathrm{T}$ cells from WT mice stimulated with anti-CD3 and anti-CD28 antibodies ( $\alpha$-CD3/28). (D-F) Immunoblot analysis using whole-cell lysates (WL) and nuclear (NF) and cytoplasmic (CF) fractions of CD8 ${ }^{+} T$ cells from WT mice stimulated with anti-CD3 and anti-CD28 antibodies (D), CD8 $8^{+}$cells from WT mice treated with $0.2 \mathrm{mM} \mathrm{H}_{2} \mathrm{O}_{2}$ for 1 hour $(E)$, and $C D 8^{+} \mathrm{T}$ cells from WT mice stimulated with anti-CD3 and anti-CD28 antibodies plus $10 \mathrm{mM}$ NAC for 1 hour (F). (G and $\mathbf{H})$ Histogram shows the MFI of ROS (G) and quantification of the MFI of ROS ( $\mathbf{H}, n=7)$ in CD8 ${ }^{+}$T cells from the spleens (Spl) and tumors (TIL) of tumor-bearing mice (day 7 after injection of tumors with MC38 cells). (I and J) Immunoblot analysis using CD8 ${ }^{+} T$ cells from the spleens and tumors of tumor-bearing mice (I) and tumor-infiltrating CD8 ${ }^{+}$T cells treated with $10 \mathrm{mM} \mathrm{NAC}$ for 1 hour (J). (K and $\left.\mathbf{L}\right)$ Histogram shows the MFI of ROS (K) and quantification of the MFI of ROS (L, $n=4)$ in CD8 ${ }^{+}$T cells from patient-derived PBMCs and CRC tissues. (M and $\left.\mathbf{N}\right)$ Immunoblot analysis of the indicated proteins in CD8 ${ }^{+}$T cells from patient-derived PBMCs and CRC tissues (M) and CD8 ${ }^{+}$T cells from CRC tissues treated with 10 mM NAC for 1 hour (N). Representative data are shown from $2(\mathbf{A}, \mathbf{M}$, and $\mathbf{N})$ and $3(\mathbf{C}-\mathbf{F}, \mathbf{I}$, and $\mathbf{J})$ independent experiments. ${ }^{*} P<0.05$ and ${ }^{* *} P<0.01$, by Student's $t$ test $(\mathbf{B}, \mathbf{H}$ and $\mathbf{L})$.

cells (Figure 4E). Moreover, SENP7-deficient CD8 ${ }^{+} \mathrm{T}$ cells displayed decreased oxidative phosphorylation (OXPHOS) rates at maximum capacity (Figure 4F). Hence, SENP7 promotes activation of PI3K/ mTOR signaling to sustain $\mathrm{CD}^{+} \mathrm{T}$ cell glycolysis and OXPHOS.

SENP7-dependent reduction in PTEN controls CD8 ${ }^{+} T$ cell metabolism and function. Our results revealed that SENP7 deficiency inhibited phosphorylation of AKT at Thr308 in CD8 ${ }^{+} \mathrm{T}$ cells (Figure $5 \mathrm{~A}$ ), indicating that the upstream pathway of PI3K/mTOR signaling is regulated by SENP7. However, PI3K activity was comparable in WT and KO CD8 ${ }^{+} \mathrm{T}$ cells (Figure $5 \mathrm{~B}$ ). Since PTEN serves as the main negative regulator of PI3K (35), we next examined the activity of PTEN. Interestingly, TCR and CD28 stimulation led to a reduction of PTEN protein expression in $\mathrm{WT} \mathrm{CD}^{+} \mathrm{T}$ cells (Figure 5C). Notably, SENP7 deficiency strongly prevented the $\mathrm{T}$ cell receptor- (TCR-) and CD28-induced reduction in PTEN expression (Figure 5C). To validate the contribution of PTEN activity to the observed phenotypes of SENP7-deficient CD8 ${ }^{+} \mathrm{T}$ cells, we crossed Senp $7^{f / / f l} C d 4$-Cre (KO) mice with Pten ${ }^{f / f l}$ mice to generate Senp $7^{f / f l}$ $\mathrm{Pten}^{\mathrm{f} / \mathrm{fl}} \mathrm{Cd}$-Cre (double-KO [DKO]) mice. Compared with stimulated Senp $7^{f / f l} C d 4$-Cre (KO) $\mathrm{CD}^{+} \mathrm{T}$ cells, stimulated DKO CD8 ${ }^{+}$ $\mathrm{T}$ cells showed markedly enhanced mTORC1 activity (Figure 5D). Ki-67 expression and the proliferation of activated SENP7-deficient $\mathrm{CD}^{+} \mathrm{T}$ cells were considerably augmented by deletion of PTEN (Figure 5, E and F). Furthermore, the glycolytic and OXPHOS 
A

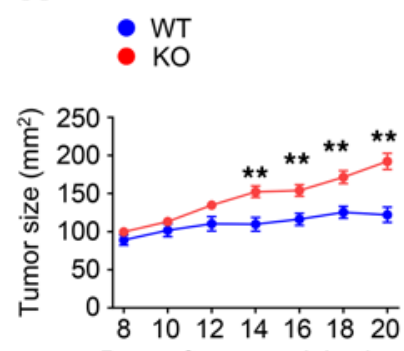

Days after tumor injection
B

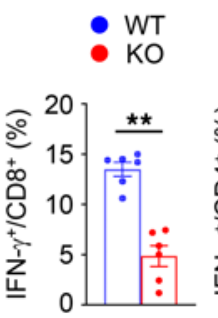

C

- WT

- $\mathrm{KO}$
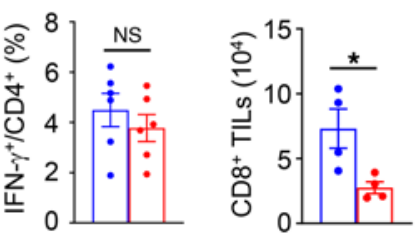

D

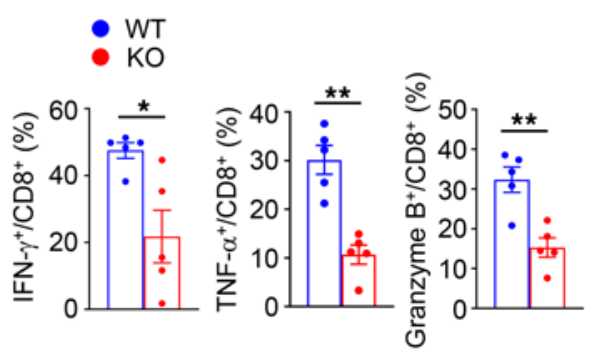

E

TILs

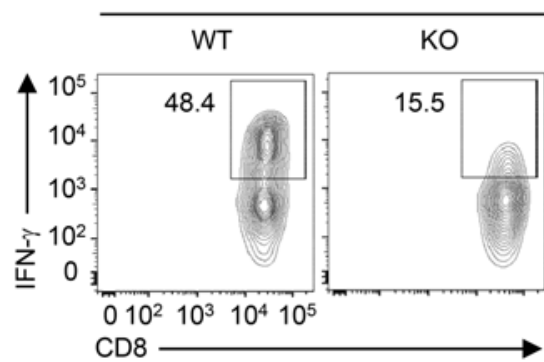

$\mathbf{F}$
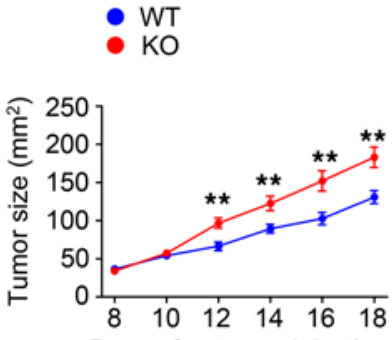

Days after tumor injection
G
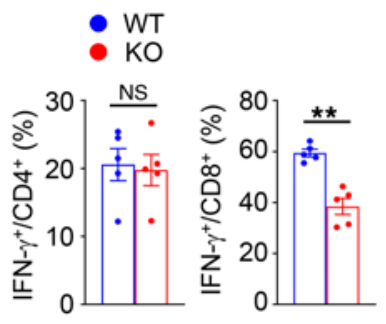

H
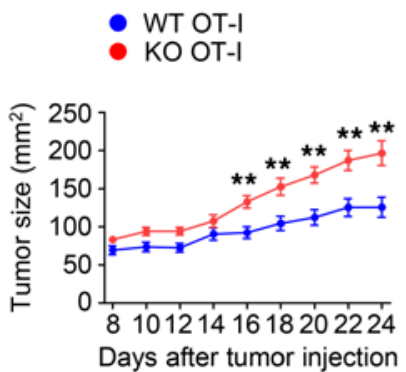

WT

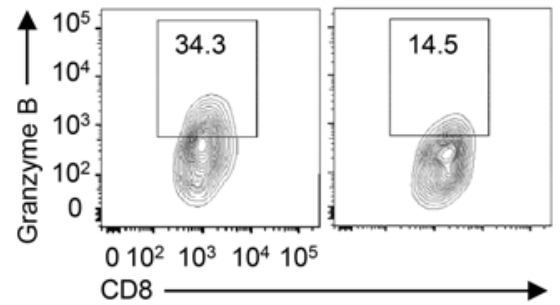

I

- WT OT-I

KO OT-I

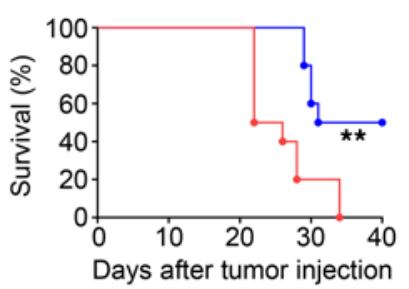

Figure 2. SENP7 ablation dampens CD8+ $T$ cell antitumor responses in vivo. (A) Tumor growth in Senp $7^{f l / f l}$ (WT) and Senp $7^{f l / f l} C d 4-C r e(K O)$ mice injected s.c. with MC38 murine colon cancer cells ( $n=10$ mice per group). (B) Flow cytometric analysis of the frequency of IFN- $\gamma$-producing CD8 ${ }^{+}$or CD4 ${ }^{+}$T cells in the draining lymph nodes of WT and KO mice injected s.c. with MC38 murine colon cancer cells (day 14, $n=6$ ). (C) CD8 ${ }^{+}$T cell numbers in tumors (TILs) of WT and KO mice injected s.c. with MC38 murine colon cancer cells (day $14, n=4$ ) were normalized to $100 \mathrm{mg}$ tumor tissue. (D and $\mathbf{E}$ ) Flow cytometric analysis of IFN- $\gamma$-producing, TNF- $\alpha$-producing, or granzyme B-producing CD8+ T cells in the tumors of WT and KO mice injected s.c. with MC38 murine colon cancer cells (day 14, $n=5$ ). The data are presented as summary graphs in $\mathbf{D}$ and as representative plots in $\mathbf{E}$. (F) Tumor growth in WT and KO mice injected s.c. with B16-F10 melanoma cells ( $n=10$ mice per group). (C) Frequency of IFN- $\gamma$-producing CD4+ ${ }^{+}$cells or CD8 ${ }^{+}$T cells in the tumors of WT and KO mice injected s.c. with B16-F10 melanoma cells (day 14, $n=5$ ). ( $(H$ and I) Tumor growth and survival curves for B6.SJL mice injected s.c. with MC38-OVA cancer cells adoptively transferred with WT OT-I or Senp $7^{f / f l} \mathrm{Cd} 4$-Cre (KO) OT-I CD8 ${ }^{+}$T cells on day 7 after tumor cell inoculation ( $n=10$ mice per group). Representative data are shown from 3 independent experiments. Data are presented as the mean \pm SEM. ${ }^{*} P<0.05$ and ${ }^{* *} P<0.01$, by 2 -tailed Student's $t$ test (A-D and $\mathbf{F}-\mathbf{H})$ and log-rank (Mantel-Cox) test (I).

rates in activated SENP7-deficient CD8 ${ }^{+} \mathrm{T}$ cells were considerably increased by deletion of PTEN (Figure 5, G and H). Importantly, SENP7-sufficient and SENP7-deficient CD8 ${ }^{+} \mathrm{T}$ cells displayed comparable levels of $\mathbf{S 6}$ phosphorylation, Ki-67 expression, cell proliferation, glycolysis, and OXPHOS in the absence of PTEN (Figure 5, D-H). We further reconstituted Rag1-KO mice with WT, Senp 7f/ll $^{\text {R }}$ Cd4-Cre (KO), Pten ${ }^{f / f l} C d 4$-Cre (Pten-KO), or Pten ${ }^{f / f l}$ Senp ${ }^{7 / / f l} C d 4$ Cre (DKO) $\mathrm{CD} 8^{+} \mathrm{T}$ cells along with an equal number of WT CD4 $4^{+}$ $\mathrm{T}$ cells. After MC38 tumor challenge, Rag1-KO mice reconstituted with PTEN-deficient $\mathrm{CD} 8^{+} \mathrm{T}$ cells plus WT $\mathrm{CD} 4^{+} \mathrm{T}$ cells had enhanced antitumor immunity (Figure 5, I and J). Importantly, the tumor size and frequency of IFN- $\gamma$-producing $\mathrm{CD} 8^{+} \mathrm{T}$ cells in tumors were similar between the recipients of $\mathrm{DKO} C D 8^{+} \mathrm{T}$ cells plus WT CD4 $4^{+} \mathrm{T}$ cells and the recipients of Pten-KO CD8 ${ }^{+} \mathrm{T}$ cells plus WT CD $4^{+} \mathrm{T}$ cells (Figure 5, I and J). These results suggest that a SENP7-dependent reduction in PTEN maintains $\mathrm{CD} 8^{+} \mathrm{T}$ cell metabolic fitness and effector function.

SENP7-mediated PTEN deSUMOylation facilitates PTEN degradation. We next sought to determine whether SENP7 mediates deSUMOylation to regulate PTEN activity. SENP7 was found to interact with PTEN in $\mathrm{CD} 8^{+} \mathrm{T}$ cells (Supplemental Figure 4A). Transfection of human embryonic kidney 293T (HEK293T) cells with the corresponding constructs further confirmed the interaction between SENP7 and PTEN (Supplemental Figure 4B). When we coexpressed PTEN with SENPT, PTEN SUMOylation was dramatically inhibited, but this effect did not occur with coexpression 


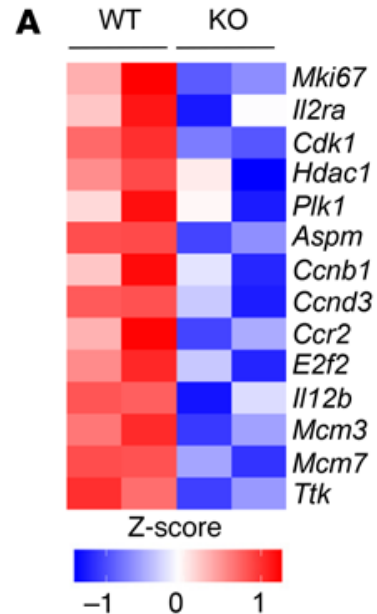

B

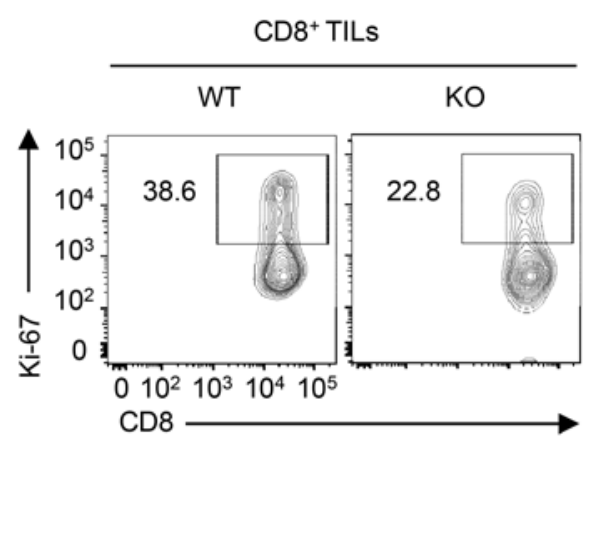

C

- WT

$\circ \mathrm{KO}$

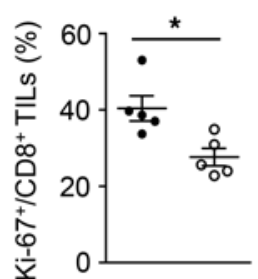

- WT

$\circ \mathrm{KO}$

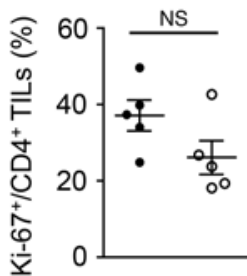

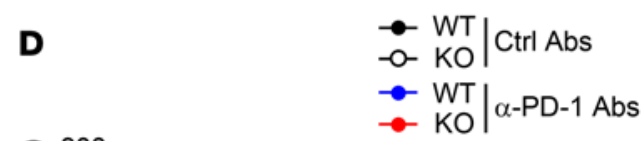
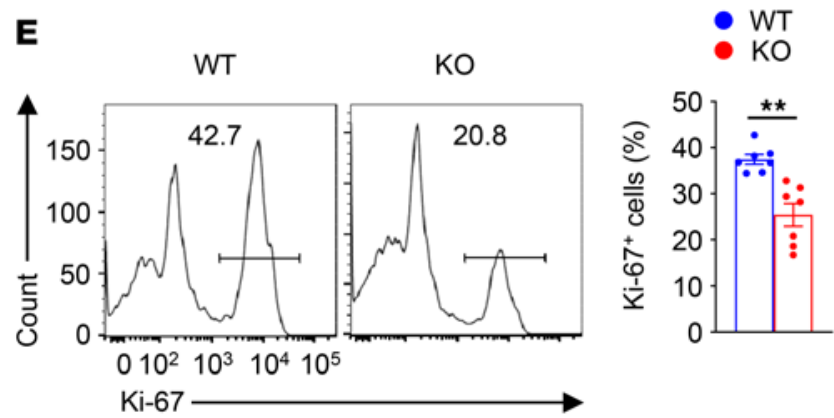

$\mathbf{F}$
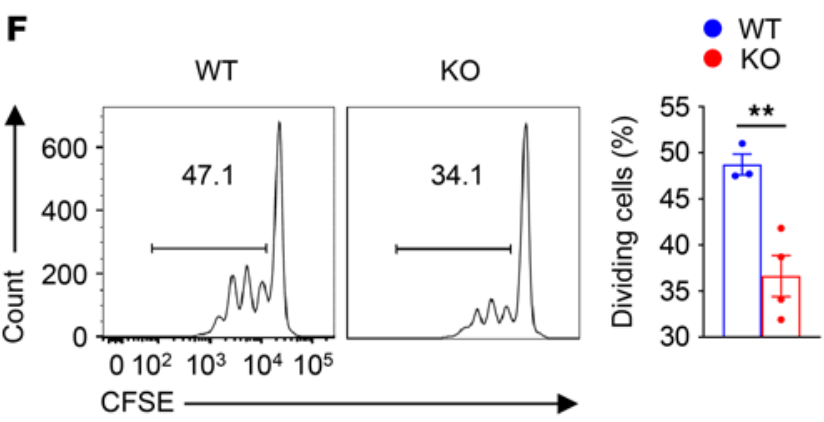

G
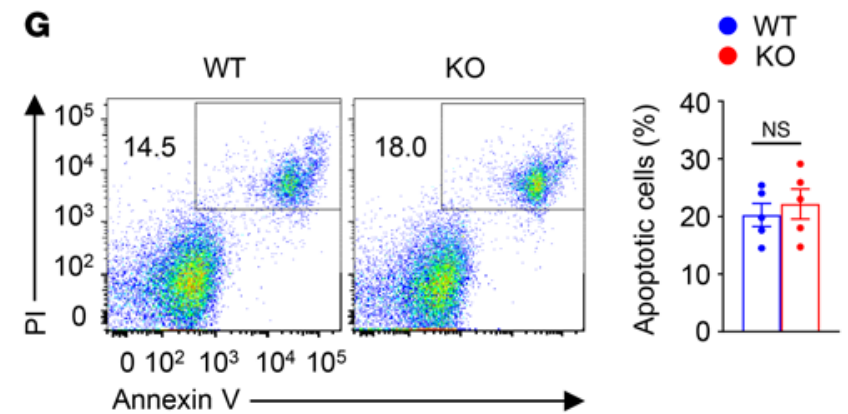

Figure 3. SENP7 is indispensable for CD8 ${ }^{+} \mathbf{T}$ cell proliferation in vivo and in vitro. (A) Heatmap of downregulated genes associated with T cell proliferation in tumor-infiltrating WT and SENP7-deficient CD8 ${ }^{+} \mathrm{T}$ cells. CD8 ${ }^{+} \mathrm{T}$ cells were isolated from tumor-bearing WT and KO mice on day 7 after injection of tumors with MC38 murine colon cancer cells. (B and C) Flow cytometric analysis of the frequency of Ki-67+ tumor-infiltrating CD8 ${ }^{+} \mathrm{T}$ cells (B) and CD4 ${ }^{+} \mathrm{T}$ cells (C) from WT and KO mice injected s.c. with MC38 murine colon cancer cells (day 14, $n=5$ ). (D) Tumor growth in WT and KO mice injected with MC38 colon cancer cells $(n=6)$ followed by i.p. injection with $50 \mu$ g anti-PD-1 antibody or control antibody (Ctrl) on days 7, 10, and 13. (E) Flow cytometric analysis of the frequency of Ki-67+ WT and KO CD8 ${ }^{+}$T cells stimulated with anti-CD3 and anti-CD28 antibodies for 2 days $(n=7)$. (F) Flow cytometric analysis of the division of WT and KO CD8 ${ }^{+}$T cells. Naive WT and KO CD8 ${ }^{+}$T cells labeled with CFSE were stimulated for 72 hours with antibodies against CD3 and CD28.

(G) Flow cytometric analysis of apoptotic WT and KO CD8 ${ }^{+}$T cells stimulated with anti-CD3 and anti-CD28 antibodies for 1 day $(n=5)$. Data are representative of 3 or more independent experiments and are presented as the mean \pm SEM. ${ }^{*} P<0.05$ and ${ }^{*} P<0.01$, by 2 -way ANOVA with Geisser-Greenhouse correction (D) and 2-tailed Student's $t$ test (B, C, and E-C).

of the catalytically inactive SENP7 (Figure 6A). Consistent with this finding, PTEN was transiently SUMOylated in $\mathrm{KO} \mathrm{CD}^{+} \mathrm{T}$ cells upon TCR and CD28 stimulation, but this effect was not observed in WT CD $8^{+} \mathrm{T}$ cells (Figure 6, B and C), indicating that SENP7 prevents the SUMOylation of PTEN in activated $\mathrm{CD}^{+} \mathrm{T}$ cells. Treatment with a protein synthesis inhibitor, cycloheximide (CHX), led to substantial loss of PTEN in activated WT CD8 ${ }^{+} \mathrm{T}$ cells but not in activated $\mathrm{KO} \mathrm{CD}^{+} \mathrm{T}$ cells (Figure 6D), indicating that SENP7 regulates PTEN degradation. Because K48-linked ubiquitin chains are the primary signal for protein degradation (36), we next examined the level of K48-linked ubiquitination (K48Ub) of PTEN in activated $\mathrm{CD} 8^{+} \mathrm{T}$ cells. Blocking protein degradation with a proteasome inhibitor, MG132, resulted in accumulation of PTEN in WT CD8 ${ }^{+}$ $\mathrm{T}$ cells, similar to that observed in SENP7-deficient $\mathrm{CD}^{+} \mathrm{T}$ cells (Figure 6E). In this condition, we observed abundant K48-linked ubiquitination of PTEN in activated WT $\mathrm{CD} 8^{+} \mathrm{T}$ cells (Figure $6 \mathrm{E}$ ), and this modification is likely responsible for TCR- and CD28induced PTEN degradation. However, this phenomenon did not occur in activated SENP7-deficient CD8 ${ }^{+} \mathrm{T}$ cells (Figure 6E), indicating that dramatically increased PTEN SUMOylation inhibits 


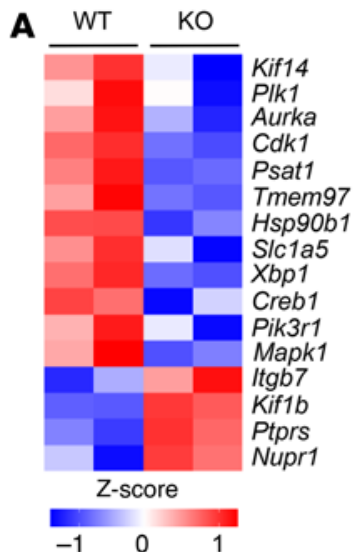

$\mathbf{E}$

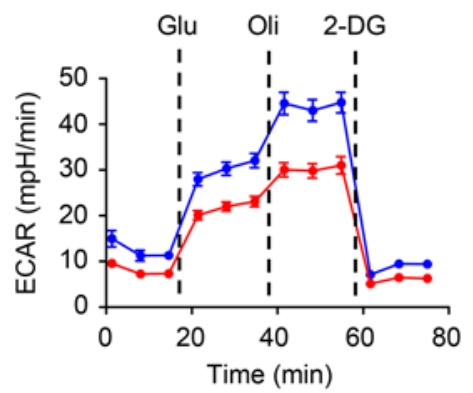

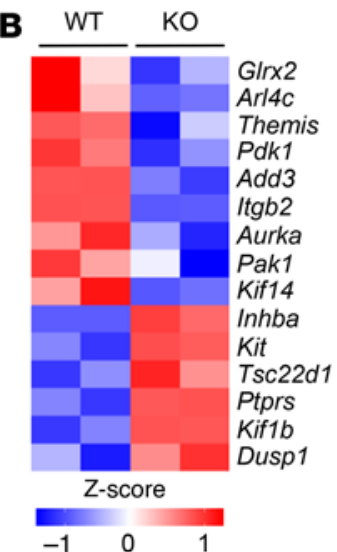

WT

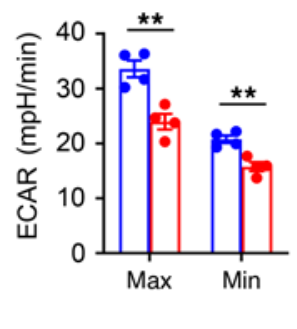

C

D

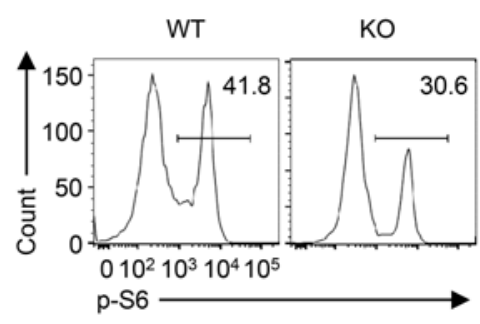

$\mathbf{F}$

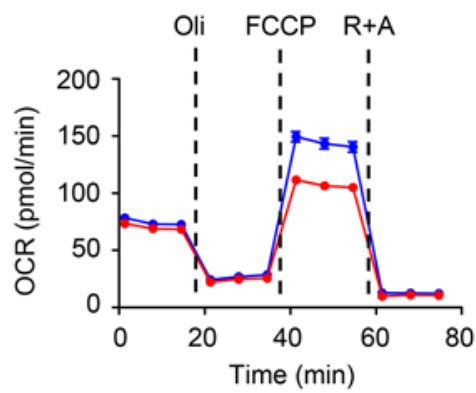

- WT

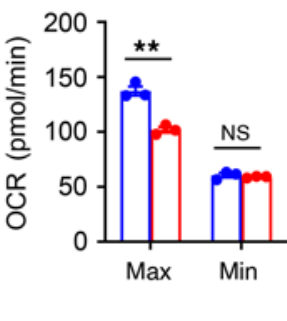

Figure 4. SENP7 promotes glycolysis and OXPHOS of CD8+ $\mathbf{T}$ cells. (A) RNA-Seq analysis using WT and KO CD8 ${ }^{+} \mathrm{T}$ cells isolated from the tumors of mice injected s.c. with MC38 colon cancer cells (day 7 after injection). Heatmap shows PI3K/mTOR signaling pathway-related genes. (B) RNA-Seq analysis using naive WT and KO CD8 ${ }^{+}$T cells stimulated with anti-CD3 and anti-CD28 antibodies for 8 hours. Heatmap shows PI3K/mTOR signaling pathway-related genes. (C) Immunoblot analysis of the indicated proteins in WT and KO CD8 ${ }^{+}$T cells stimulated with anti-CD3 and anti-CD28 antibodies for the indicated durations. (D) Flow cytometric analysis of p-S6 expression in WT and KO CD8 ${ }^{+} T$ cells stimulated with anti-CD3 and anti-CD28 antibodies for 2 hours. (E) ECAR of WT and KO naive CD8 ${ }^{+}$T cells stimulated for 6 hours with antibodies against CD3 and CD28 under minimum (Min) or maximum (Max) conditions with the addition of different reagents $(n=4)$. (F) OCR of WT and KO naive CD8 ${ }^{+}$T cells stimulated for 6 hours with antibodies against CD3 and CD28 under minimum or maximum conditions with the addition of different reagents $(n=3)$. Data are representative of 3 independent experiments and are presented as the mean $\pm S E M$. ${ }^{* *} P<0.01$, by Student's $t$ test. Glu, glucose; Oli, oligomycin; R+A, rotenone/antimycin $A$.

the K48-linked ubiquitination and degradation of PTEN in activated SENP7-deficient CD8 ${ }^{+}$T cells. On the other hand, loss of SENP7 did not change the SUMOylation status or stability of PTEN in activated $\mathrm{CD}^{+} \mathrm{T}$ cells (Supplemental Figure $4 \mathrm{C}$ ), which is most likely due to the low levels of SENP7 (Figure 1C).

We further examined the potential connection of TCR-triggered PTEN degradation with TCR-induced SENP7 cytosolic translocation. In response to treatment with leptomycin B (LMB), an inhibitor of nuclear export, TCR signal-induced SENP7 cytosolic translocation was largely blocked in WT CD8 ${ }^{+} \mathrm{T}$ cells (Figure $6 \mathrm{~F}$ ). We observed abundant SUMOylation of PTEN in the cytoplasm but not the nucleus of activated SENP7-deficient CD8 ${ }^{+} \mathrm{T}$ cells (Figure 6, G and H). Notably, PTEN stability was greatly restored in activated WT CD8 ${ }^{+}$ $\mathrm{T}$ cells after the treatment with LMB (Figure 6I). Moreover, LMB treatment markedly increased the SUMOylation and reduced the K48-linked ubiquitination of PTEN in the cytoplasm but not the nucleus of activated WT CD8 ${ }^{+}$T cells (Figure 6, J and K). By contrast, LMB treatment did not alter the stability, SUMOylation, or K48linked ubiquitination of PTEN in activated SENP7-deficient $\mathrm{CD}^{+} \mathrm{T}$ cells (Supplemental Figure 4, D-F). These data indicate that cytosolic SENP7 mediates PTEN deSUMOylation to facilitate the K48-linked ubiquitination and degradation of PTEN in activated CD8 ${ }^{+} \mathrm{T}$ cells.
ROS are required for SENP7-dependent $C D 8^{+} T$ cell metabolism and function. To assess whether ROS are involved in the regulation of SENP7-dependent CD8 ${ }^{+} \mathrm{T}$ cell metabolism and function, we treated WT and SENP7-deficient OT-I cells with NAC. NAC treatment substantially increased the SUMOylation and reduced the K48linked ubiquitination of PTEN in WT OT-I cells (Figure 7, A and B). Furthermore, NAC-treated WT OT-I cells exhibited decreased glycolysis and OXPHOS (Figure 7, C and D). To explore the role of ROS in $\mathrm{CD}^{+} \mathrm{T}$ cell antitumor function, WT OT-I cells stimulated with anti-CD3 and anti-CD28 antibodies plus NAC in vitro were transferred into tumor-bearing mice. NAC treatment effectively reduced the ROS levels of activated OT-I CD8 ${ }^{+} \mathrm{T}$ cells induced by anti-CD3 and anti-CD28 (Supplemental Figure 5A). Compared with untreated WT OT-I cells, NAC-treated WT OT-I cells were significantly more potent in promoting tumor progression (Figure 7E). In addition, treatment of WT OT-I cells with NAC reduced the frequencies of IFN- $\gamma^{+}$and $\mathrm{Ki}-67^{+}$OT-I cells in tumors (Figure 7F). By contrast, NAC treatment did not alter the SUMOylation or K48-linked ubiquitination of PTEN, glycolysis, or OXPHOS in activated SENP7-deficient OT-I cells (Supplemental Figure 5, B and C, and Figure 7, C and D). Moreover, mice injected with SENP7-deficient OT-I cells treated or not with NAC displayed no apparent differences 
A

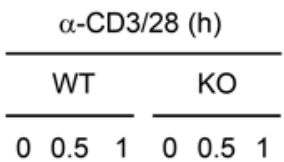

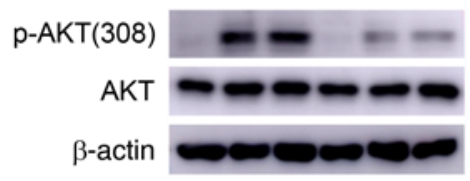

D
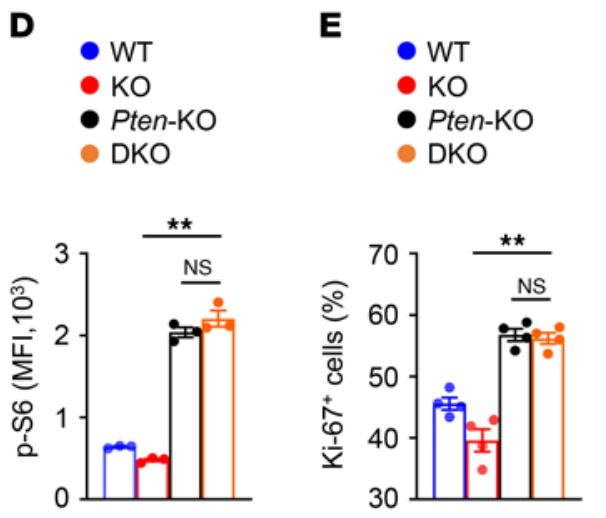

B

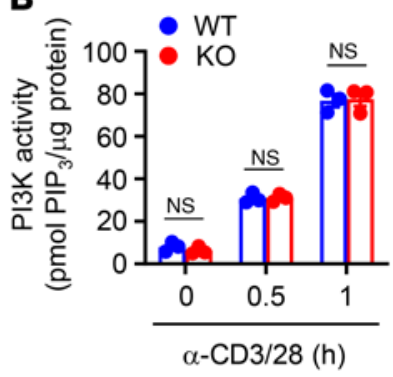

C

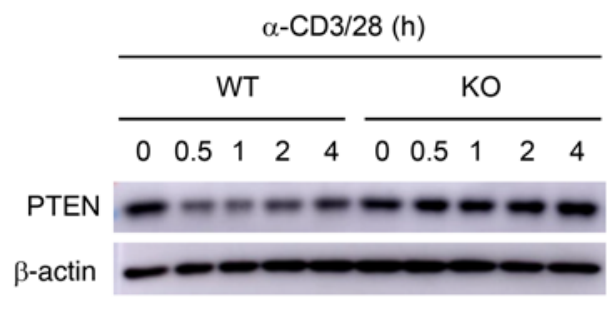

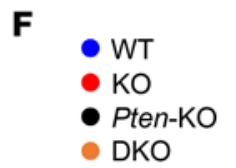

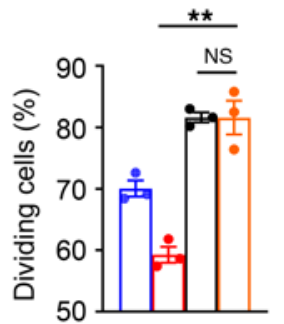

G
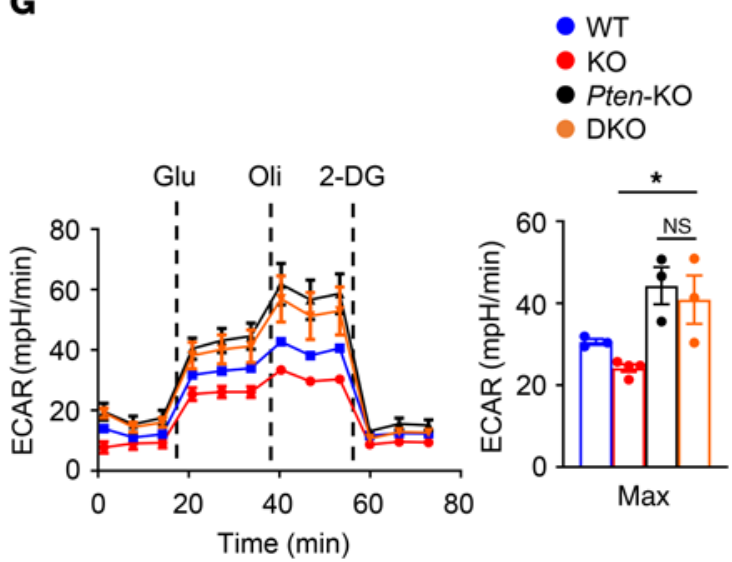

H

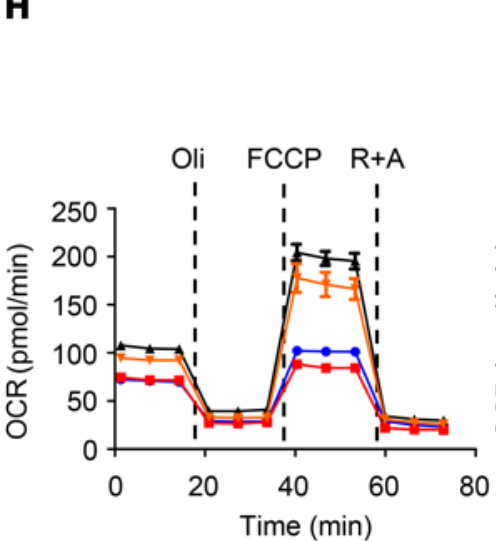

I
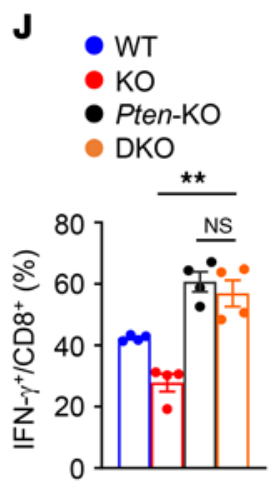

Figure 5. SENP7-dependent reduction of PTEN controls CD8 ${ }^{+}$T cell metabolism and function. (A) Immunoblot analysis of the indicated proteins in WT and $K O$ CD8 $^{+} T$ cells after stimulation with anti-CD3 and anti-CD28 antibodies. (B) PI3K activity in WT and KO CD8 ${ }^{+}$T cells stimulated with anti-CD3 and anti-CD28 antibodies was analyzed using an Echelon kit $(n=3)$. PIP ${ }_{3}$, phosphatidylinositol $(3,4,5)$-trisphosphate. (C) Immunoblot analysis of the indicated proteins in WT and KO CD8 ${ }^{+}$T cells after stimulation with anti-CD3 and anti-CD28 antibodies. (D and E) Flow cytometric analysis of $p-S 6$ (D) and Ki-67 (E) expression in WT, Senp $7^{f l / f l}$ Cd4-Cre (KO), Pten ${ }^{f / f l}$ Cd4-Cre (Pten-KO), and Pten fl/fl Senp $7^{f / f l}$ Cd4-Cre (DKO) CD8 ${ }^{+}$T cells stimulated with anti-CD3 and antiCD28 antibodies for 2 hours $(\mathbf{D}, n=3)$ or 2 days $(\mathbf{E}, n=4)$. (F) Flow cytometric analysis of the division of WT, KO, Pten-KO, and DKO CD8 $8^{+}$T cells labeled with CFSE upon stimulation with anti-CD3 and anti-CD28 antibodies for 72 hours $(n=3)$. (G and $\mathbf{H})$ ECAR $(\mathbf{C})$ and OCR $(\mathbf{H})$ of WT, KO, Pten-KO, and DKO CD8 ${ }^{+} \mathrm{T}$ cells stimulated for 6 hours with anti-CD3 and anti-CD28 antibodies. (I) Tumor growth of MC38 tumor-bearing Rag1-KO mice injected with $2 \times 10^{6}$ WT, KO, Pten-KO, or DKO CD8 ${ }^{+}$T cells along with $2 \times 10^{6}$ WT CD4 ${ }^{+}$T cells on day 7 after tumor cell inoculation $(n=10)$. (J) Flow cytometric analysis of IFN- $\gamma$-producing CD8 ${ }^{+} \mathrm{T}$ cells in the tumors of Rag1-KO mice from I (day 12, $n=4$ ). Data shown are representative of 3 independent experiments and are presented as the mean \pm SEM. ${ }^{*} P<0.05$ and ${ }^{*} P<0.01$, by 1 -way ANOVA with Tukey's multiple-comparison test (D-H and $\left.\mathbf{J}\right) ; 2$-way ANOVA with Geisser-Greenhouse correction (I); and 2-tailed Student's $t$ test (B).

in tumor growth or the frequencies of tumor-infiltrating IFN- $\gamma^{+}$and Ki-67+ OT-I cells (Supplemental Figure 5, D and E). These results suggest that ROS inhibition perturbs tumor-infiltrating $\mathrm{CD}^{+} \mathrm{T}$ cell metabolism and function in an SENP7-dependent manner.

Given the potential importance of ROS in $\mathrm{CD}^{+} \mathrm{T}$ cell antitumor function, we analyzed the relationship between ROS and tumor- infiltrating $\mathrm{CD}^{+} \mathrm{T}$ cell activity in human CRC. Indeed, we found that NAC treatment restored PTEN stability in $\mathrm{CD}^{+} \mathrm{T}$ cells from CRC tissue (Figure 7G). Additionally, NAC-treated tumor-infiltrating $\mathrm{CD}^{+} \mathrm{T}$ cells exhibited decreased glycolysis and OXPHOS (Figure $7 \mathrm{H})$. Accordingly, NAC treatment reduced the expression of Ki-67 in CD8 ${ }^{+} \mathrm{T}$ cells from CRC tissue (Figure 7I). Collectively, these 


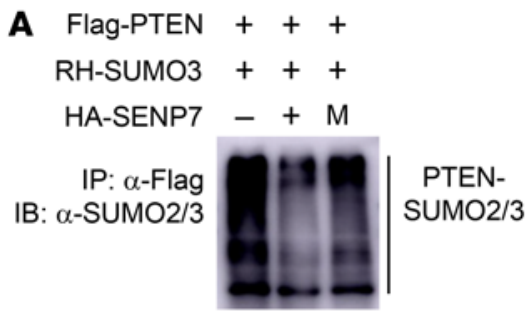

Cell lysates

HA-SENP7

Flag-PTEN

D

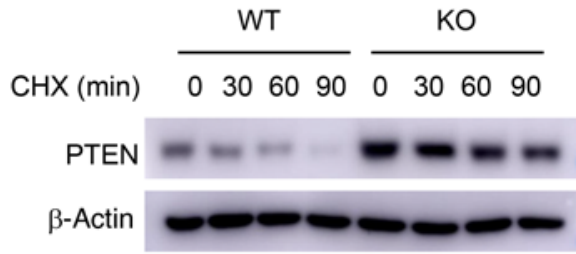

G

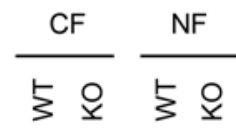

IP: $\alpha$-PTEN

IB: $\alpha$-SUMO2/3
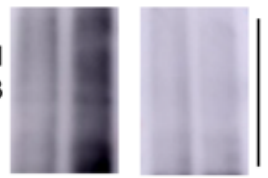

PTEN-

SUMO2/3

Cell lysates

PTEN
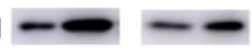

J

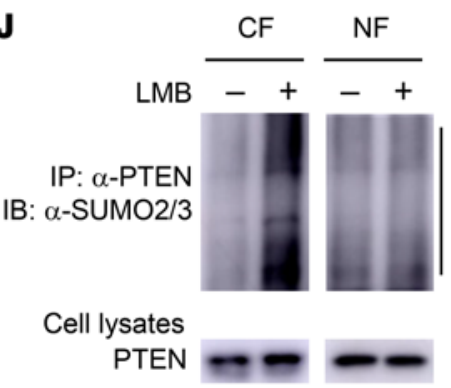

B

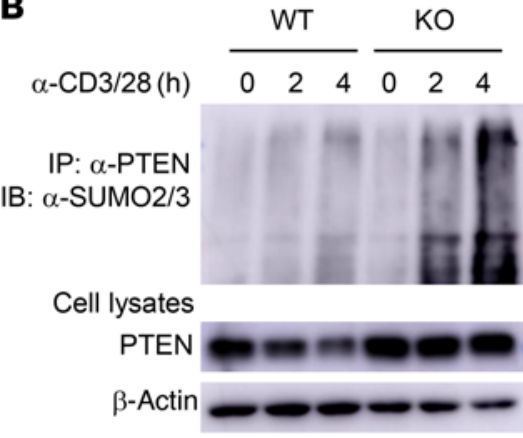

E

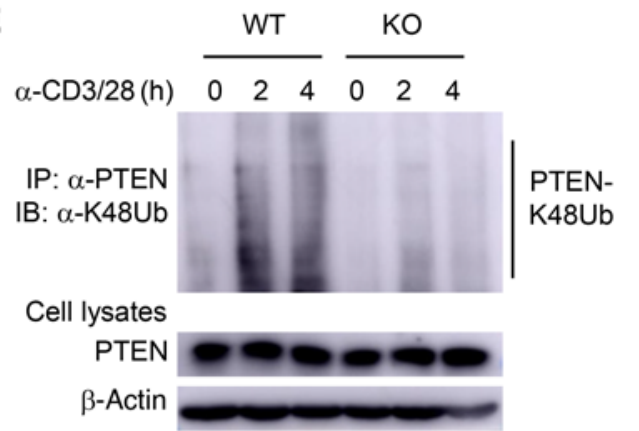

C

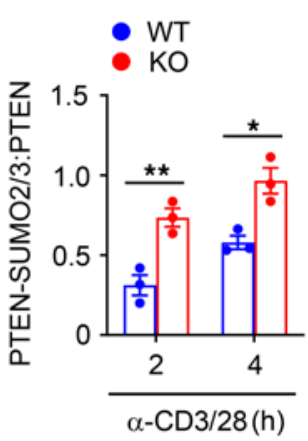

$\mathbf{F}$

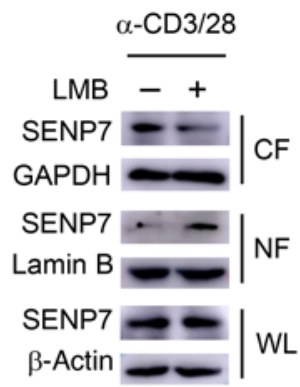

Figure 6. SENP7-mediated PTEN deSUMOylation facilitates PTEN degradation. (A) HEK293T cells cotransfected with Flag-tagged PTEN, Ubc9, and RH-SUMO3 in the presence of WT or catalytically inactive (C979S) mutant (M) SENP7 were immunoprecipitated with anti-Flag antibody and assessed by immunoblotting (IB) with anti-SUMO2/3. HA, hemagglutinin; RH, arginine(R)-glycine(C)-serine(S)-histidine. (B) PTEN SUMOylation assays were performed by immunoprecipitating PTEN under denaturing conditions followed by detection of SUMOylated PTEN using anti-SUMO2/3 antibodies in CD8 ${ }^{+}$ T cells stimulated with anti-CD3 and anti-CD28 antibodies. (C) Quantifications of PTEN-SUM02/3:PTEN levels in CD8 ${ }^{+}$T cells stimulated with anti-CD3 and anti-CD28 antibodies $(n=3)$. (D) Immunoblot analysis of the indicated proteins in CD8 ${ }^{+} T$ cells stimulated with anti-CD3 and anti-CD28 antibodies for 1 hour following incubation with $\mathrm{CHX}(50 \mu \mathrm{g} / \mathrm{mL})$ for the indicated durations. (E) PTEN ubiquitination assays in CD8 ${ }^{+}$T cells stimulated with anti-CD3 and anti-CD28 antibodies in the presence of MG132. (F) Immunoblot analysis of the indicated proteins in CD8 ${ }^{+}$T cells from WT mice stimulated with anti-CD3 and anti-CD28 antibodies plus LMB for 2 hours. (G) PTEN SUMOylation assays using nuclear and cytoplasmic fractions of WT and KO CD8 ${ }^{+}$T cells stimulated with anti-CD3 and anti-CD28 antibodies for 2 hours. (H) Quantification of PTEN-SUMO2/3:PTEN levels in the cytoplasmic fractions of WT and KO $\mathrm{CD}^{+} \mathrm{T}$ cells stimulated with anti-CD3 and anti-CD28 antibodies for 2 hours $(n=4)$. (I) Immunoblot analysis of the indicated proteins in CD8 ${ }^{+}$T cells from WT mice stimulated with anti-CD3 and anti-CD28 antibodies plus LMB for 0, 2, and 4 hours. (J and K) PTEN SUMOylation (J) and ubiquitination (K) assays using nuclear and cytoplasmic fractions of CD8 ${ }^{+} T$ cells from WT mice stimulated with anti-CD3 and anti-CD28 antibodies plus LMB for 2 hours. Data are representative of 3 independent experiments and presented as the mean \pm SEM. ${ }^{*} P<0.05$ and ${ }^{* *} P<0.01$, by Student's $t$ test. 
A
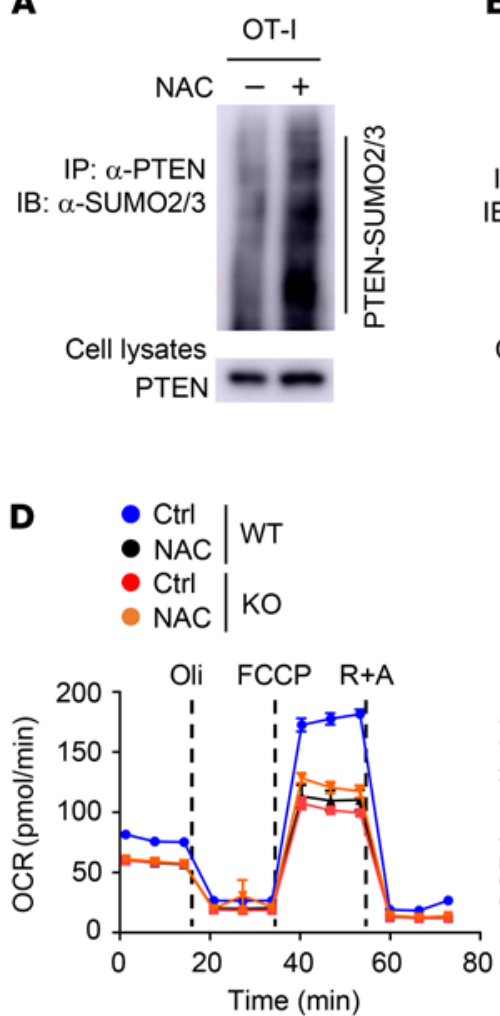

G

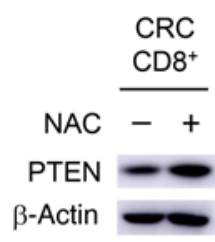

H $\quad$ - Ctrl
B

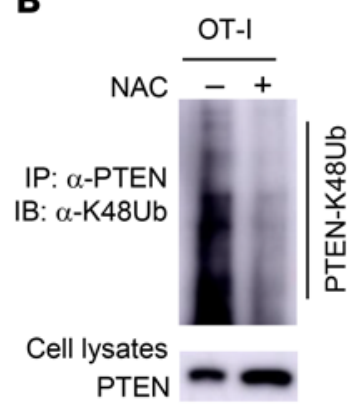

$\mathbf{E}$
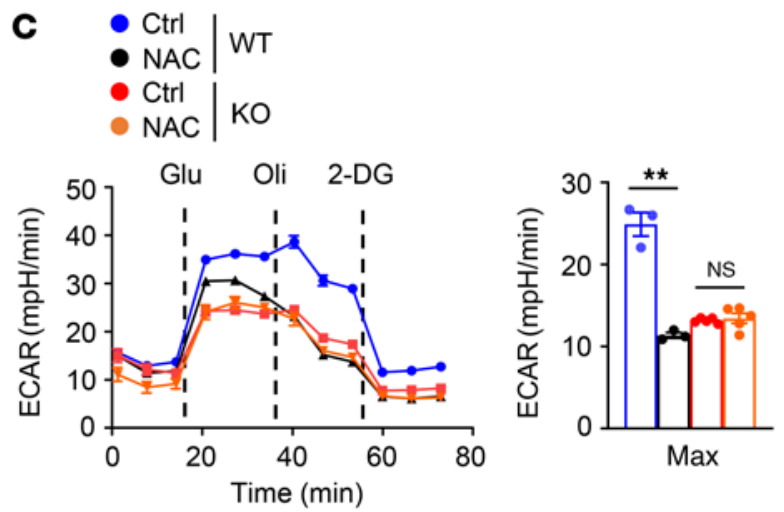
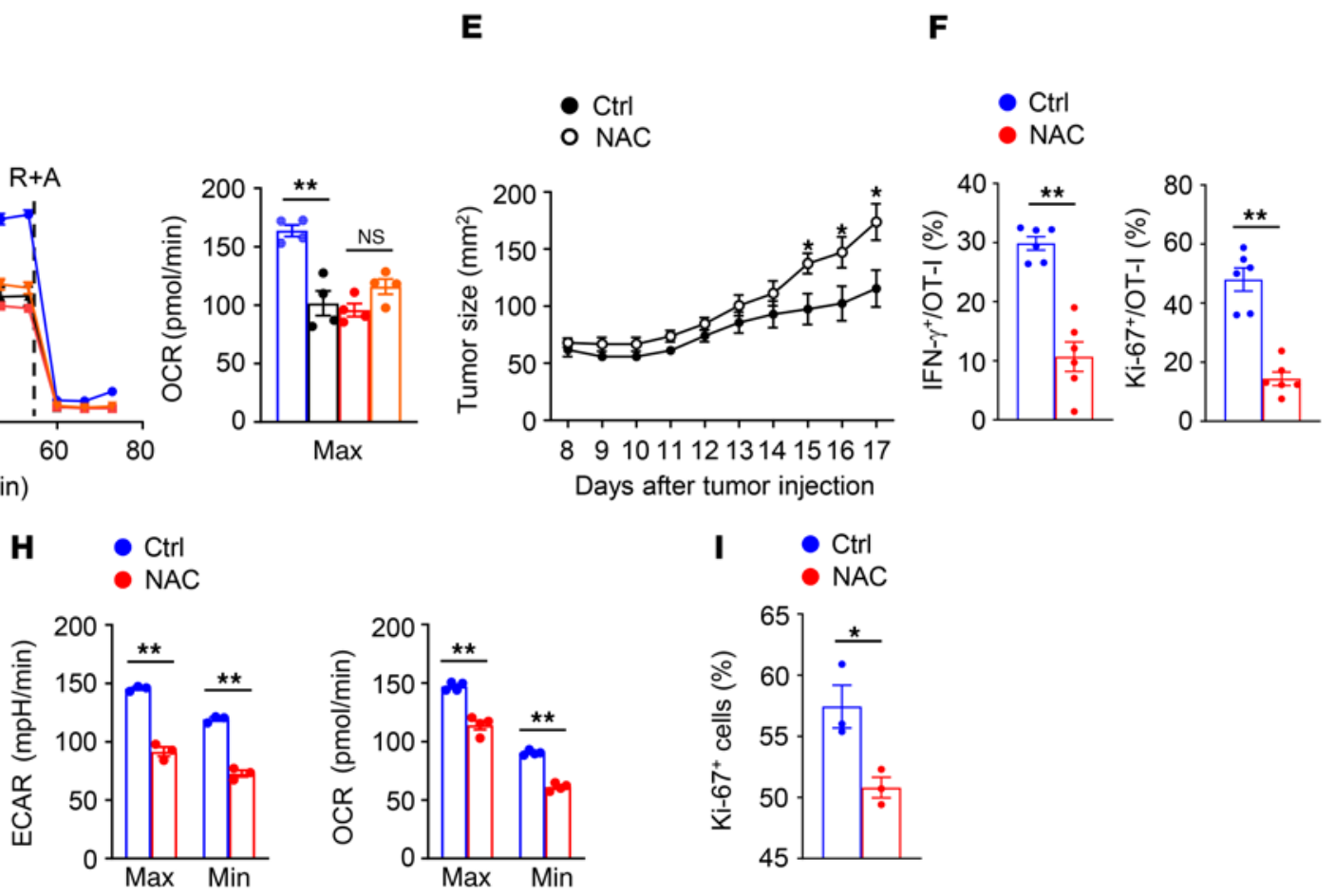

Figure 7. ROS involve SENP7-dependent CD8+ T cell metabolism and function. (A and B) PTEN SUMOylation (A) and ubiquitination (B) assays using WT OT-I cells stimulated with anti-CD3 and anti-CD28 antibodies plus NAC for 2 hours. (C and D) ECAR (C) and OCR (D) of WT and KO OT-I cells stimulated with anti-CD3 and anti-CD28 antibodies plus NAC for 6 hours. (E) Tumor growth of MC38-OVA tumor-bearing WT mice (day 6 after tumor cell inoculation) injected i.v. with WT OT-I cells stimulated with anti-CD3 and anti-CD28 antibodies plus 10 mM NAC for 8 hours in vitro (control: $n=8$; NAC: $n=9$ ). (F) Flow cytometric analysis of the frequency of IFN- $\gamma$-producing and Ki-67+ OT-I cells in the tumors of mice from $\mathbf{E}$ (day 17 after tumor injection, $n=6$ ). (C) Immunoblot analysis of the indicated proteins in CD8 ${ }^{+} T$ cells from CRC samples incubated in vitro for 2 hours in complete media containing $10 \mathrm{mM} N A C$. (H) ECAR and OCR of CD8 ${ }^{+}$T cells from CRC samples incubated in vitro for 2 hours in complete media containing 10 mM NAC. (I) Flow cytometric analysis of the frequency of $\mathrm{Ki}-67^{+} \mathrm{CD} 8^{+} \mathrm{T}$ cells from CRC samples incubated in vitro for 6 hours in complete media containing $10 \mathrm{mM} \mathrm{NAC}(n=3)$. Data are representative of 3 independent experiments and presented as the mean \pm SEM. ${ }^{*} P<0.05$ and ${ }^{*} P<0.01$, by 1 -way ANOVA with Tukey's multiple-comparison test (C and D) and 2-tailed Student's $t$ test (E, F, H, and I).

results suggest that ROS are required for SENP7-dependent the metabolic fitness and antitumor function of $\mathrm{CD}^{+} \mathrm{T}$ cells.

\section{Discussion}

In this work, we revealed a crucial role of oxidative stress in CD8 ${ }^{+}$ $\mathrm{T}$ cell metabolic fitness and effector function by manipulating SENP7 activity. SENP7 was required for the metabolic reprogramming of $\mathrm{CD}^{+} \mathrm{T}$ cells, which is critical for their proliferation in vitro and antitumor immune responses in vivo. In response to TCRinduced ROS, SENP7 rapidly translocated from the nucleus to the cytoplasm to mediate PTEN deSUMOylation and in turn promoted
PTEN degradation, thereby maintaining the $\mathrm{CD} 8^{+} \mathrm{T}$ cell metabolic state. Importantly, SENP7 sensed ROS to suppress PTEN-dependent metabolic dysfunction and functional decline in human CRC samples. These findings not only suggest that SENP7 sustains the metabolic and functional integrity of $\mathrm{CD} 8^{+} \mathrm{T}$ cells but also highlight what we believe to be a new link between oxidative stress and $\mathrm{CD}^{+} \mathrm{T}$ cell metabolic reprogramming.

Oxidative stress is mediated by ROS and involves $\mathrm{CD}^{+} \mathrm{T}$ cell activation, expansion, and function (18). ROS elevation upon TCR stimulation is essential for antigen-specific $\mathrm{T}$ cell expansion in vivo (18). Impaired oxidative metabolism with reduced ROS production 
results in antitumor $\mathrm{T}$ cell dysfunction (20). We obtained strong evidence that $\mathrm{T}$ cell-intrinsic ROS are indispensable for $\mathrm{CD}^{+} \mathrm{T}$ cell metabolic fitness and effector functions. Pharmacologically targeting of ROS disrupted the metabolism and impaired the antitumor functions of tumor-infiltrating $\mathrm{CD} 8^{+} \mathrm{T}$ cells. At a minimum, our findings suggest that SENP7 serves as a ROS sensor to facilitate $\mathrm{CD}^{+} \mathrm{T}$ cell metabolic reprogramming during naive-to-effector differentiation. Notably, aberrantly increased mitochondrial ROS in $\mathrm{CD}^{+} \mathrm{T}$ cells under hypoxia drive an exhaustion-like dysfunctional program (21). In addition, abnormal ROS accumulation induced by G6pd mutation disrupts $\mathrm{CD}^{+} \mathrm{T}$ cell responses to tumorigenesis (33). Physiological levels of ROS are likely critical for $\mathrm{CD}^{+} \mathrm{T}$ cell metabolic fitness and effector function, whereas excessive ROS production can cause $\mathrm{CD}^{+} \mathrm{T}$ cell dysfunction. Whether different $\mathrm{CD}^{+} \mathrm{T}$ cell subtypes have different intensities of ROS production and how SENP7 senses these different ROS levels to shape $\mathrm{CD} 8^{+} \mathrm{T}$ cell identity remain to be clarified.

Activation of $\mathrm{PI} 3 \mathrm{~K} / \mathrm{mTOR}$ signaling in early-phase $\mathrm{T}$ cell activation involves metabolic reprogramming $(9,37,38)$. PTEN acts as an inhibitor of PI3K/mTOR signaling, and its TCR signal-induced translocation to the plasma membrane is required for its phosphatase activity to terminate PI3K/mTOR signaling (34). Our previous results revealed that the lipid kinase AGK rapidly phosphorylates PTEN at Ser380, Thr382, and Thr383 during early T cell activation, thereby attenuating PTEN activity and preventing premature signal termination in $\mathrm{CD}^{+}$T cells (34). Subsequently, PTEN undergoes ubiquitination and proteasomal degradation upon TCR stimulation. Here, we provide in-depth insight into the molecular mechanism underlying PTEN degradation-mediated metabolic control in activated $\mathrm{CD}^{+} \mathrm{T}$ cells. Our current results showed that TCR-induced SENP7 cytosolic translocation facilitated the deSUMOylation, ubiquitination, and degradation of PTEN, thus allowing sustained activation of PI3K/mTOR signaling and metabolic reprogramming, thus collectively suggesting a model of SENP7-dependent PTEN restriction in activated $\mathrm{CD} 8^{+} \mathrm{T}$ cells.

SENP7 contains a conserved nuclear export sequence (NES) and regulates SUMO-mediated nuclear events, including transcription, DNA repair, and nuclear transport (30, 39-41). Although SENP7 has been reported to potentiate cGAS activation and contribute to the expansion of proinflammatory $\gamma \delta \mathrm{T}$ cells in inflammatory bowel disease, the related studies used an in vivo model of SENP7 knockdown $(31,42)$. Using Senp7-conditional KO mice, we revealed a ROS-dependent function of SENP7 in regulating $\mathrm{CD}^{+} \mathrm{T}$ cell metabolic and functional states. In resting CD ${ }^{+} \mathrm{T}$ cells, SENP7 accumulated mainly in the nucleus; however, the TCR signalinduced ROS triggered the translocation of SENP7 from the nucleus to the cytoplasm, thus limiting PTEN-dependent metabolic defects. Upon treatment with the ROS inhibitor NAC or the nuclear export inhibitor LMB, TCR signal-induced SENP7 cytosolic translocation was largely blocked, resulting in PTEN accumulation in activated $\mathrm{CD} 8^{+} \mathrm{T}$ cells. These data suggest that TCR signals initiate SENP7 cytosolic translocation in $\mathrm{CD}^{+} \mathrm{T}$ cells in a ROS-dependent manner. However, the mechanism by which ROS cause SENP7 cytosolic translocation remains to be identified.

$\mathrm{CD} 4^{+} \mathrm{T}$ cells and $\mathrm{CD} 8^{+} \mathrm{T}$ cells have unique transcriptional and metabolic programs $(43,44)$. We showed here that $C D 4^{+} \mathrm{T}$ cells had much lower expression levels of SENP7 than did CD8 ${ }^{+} \mathrm{T}$ cells. The difference in SENP7 expression levels in $\mathrm{CD}^{+} \mathrm{T}$ cells and $\mathrm{CD} 8^{+} \mathrm{T}$ cells is probably due to the different transcriptional programs. Differences in specific protein levels in $\mathrm{CD} 4^{+} \mathrm{T}$ cells and $\mathrm{CD} 8^{+} \mathrm{T}$ cells have also been reported in previous studies $(34,45)$. For example, the protein levels of ACAT2 are higher in $\mathrm{CD} 4^{+} \mathrm{T}$ cells than those in $\mathrm{CD}^{+} \mathrm{T}$ cells, whereas the protein levels of acylglycerol kinase are higher in $\mathrm{CD}^{+} \mathrm{T}$ cells than those in $\mathrm{CD} 4^{+} \mathrm{T}$ cells $(34,45)$. Therefore, the unique transcriptional program governing SENP7 expression in $\mathrm{CD}^{+} \mathrm{T}$ cells remains to be further studied.

In summary, we found that SENP7 sensed oxidative stress to sustain $\mathrm{CD}^{+} \mathrm{T}$ cell metabolic and functional fitness and was critical for effective antitumor immunity. Our results reveal an oxidative stress-sensing machinery in tumor-infiltrating $\mathrm{CD}^{+} \mathrm{T}$ cells. These findings will inform the search for oxidative stress-related agents and help to improve cancer immunotherapy outcomes.

\section{Methods}

Human samples. Patient-derived PBMCs and CRC tissues were obtained from Ruijin Hospital. Solid tumor tissues were freshly isolated and digested. Immune cells were enriched by density-gradient centrifugation. Human $\mathrm{CD} 4^{+} \mathrm{T}$ cells and $\mathrm{CD} 8^{+} \mathrm{T}$ cells were purified using CD4 MicroBeads (130-045-101, Miltenyi Biotec) and CD8 MicroBeads (130-045-201, Miltenyi Biotec), respectively.

Mice. Senp7-floxed mice were generated at the GemPharmatech using a LoxP-targeting system. The Senp7-floxed mice were crossed with $C d 4$-Cre transgenic mice (The Jackson Laboratory) to produce age-matched $\operatorname{Sen} p 7^{f / f l}$ and $\operatorname{Sen} p 7^{f / / f l} C d 4$-Cre mice. The Pten-floxed mice (The Jackson Laboratory) were crossed with $\operatorname{Senp}^{7^{f / f l} \mathrm{C}} \mathrm{Cd} 4$-Cre mice to produce Senp $7^{+/+}$Pten $^{f / f l} C d 4$-Cre (Pten-KO) and Senp $7^{f / f l}$ Pten $^{f / f l} C d 4$ Cre (DKO) mice. Rag1-KO mice, OT-I TCR-transgenic mice, OT-II TCR-transgenic mice, and B6.SJL mice were from The Jackson Laboratory. Male and female mice were sex matched and used at 6 to 10 weeks of age. Mice were maintained in a specific pathogen-free (SPF) facility at the Shanghai Jiao Tong University School of Medicine.

Cell culturing. HEK293T cells (American Type Culture Collection [ATCC]), MC38 murine colon cancer cells (Kerafast), and MC38-OVA cancer cells (provided in-house) were cultured in DMEM medium supplemented with $10 \%$ FBS and 100 units/mL penicillin-streptomycin. B16-F10 melanoma cells (ATCC) and isolated mouse and human $\mathrm{CD}^{+}$and $\mathrm{CD}^{+} \mathrm{T}$ cells were cultured in RPMI 1640 medium supplemented with $10 \%$ FBS and 100 units/mL penicillin-streptomycin.

Plasmids, antibodies, and reagents. HA-tagged mouse WT or mutant (C979S) SENP7 were subcloned into the pcDNA3.1-HA vector, and Flag-tagged mouse PTEN was subcloned into the lentiviral vector pLVX-IRES-ZsGreen1. Antibodies against SENP7 (PA5-36089; 1:500 for Western blotting [WB]) were purchased from Invitrogen (Thermo Fisher Scientific). Antibodies against GAPDH (97166T; 1:1000 for WB); S6 (2217S; 1:1000 for WB); AKT (4691P; 1:1000 for WB); phosphorylated S6 (p-S6) (4858S; 1:1000 for WB); p-AKT (Thr308) (2965S; 1:1000 for WB); p-AKT (Ser473) (4060S; 1:1000 for WB); PTEN (9559S; 1:1000 for WB); SUMO2/3 (4971S; 1:1000 for WB); APC-conjugated p-S6 (D57.2.2E); and K48-ubiquitin (12805S; 1:1000 for WB) were purchased from Cell Signaling Technology. HRP-conjugated anti-HA antibody (3F10; 1:2000 for WB) was purchased from Roche. Anti-Flag (M2, F3165; 1:2000 for WB) and anti- $\beta$-actin (A2228-100UL; 1:5000 for WB) antibodies were from MilliporeSigma. The fluorochromeconjugated antibodies against CD4 (GK1.5); CD8 (53-6.7); human 
CD8 (RPA-T8); CD44 (IM7); CD69 (H1.2F3); CD62L (MEL-14); Ki-67 (SolA15); Foxp3 (FJK-16s); IFN- $\gamma$ (XMG1.2); TCR 2 (B20.1); CM-H2DCFDA (C6827); TNF- $\alpha$ (MP6-XT22); and granzyme B (GB11) were purchased from Thermo Fisher Scientific. NAC, LMB, $N$-ethylmaleimide (NEM), and CHX were purchased from MilliporeSigma. Anti-mouse PD-1 (clone J43) and isotype control IgG (hamster IgG) antibodies were purchased from Bio X Cell.

Flow cytometry. To detect the surface markers including CD44, CD69, CD4, and CD8, cells were stained in PBS containing 2\% FBS with the indicated antibodies. Apoptotic cells were detected by FITCannexin $\mathrm{V}$ and propidium iodide (PI) staining (BD Biosciences). The fluorogenic dye H2DCFDA was used to detect ROS production. Intracellular IFN- $\gamma$ production was determined according to the manufacturer's instructions (Thermo Fisher Scientific). Intracellular Foxp3 and Ki-67 staining was performed according to the manufacturer's instructions (Thermo Fisher Scientific). For the intracellular staining of p-S6, stimulated T cells were fixed in Fix Buffer I (BD) for 10 minutes and then incubated in cold Perm Buffer III (BD) for 20 minutes, followed by antibody staining and flow cytometric analysis.

$T$ cell isolation and stimulation. Naive $\mathrm{CD} 4^{+}$and $\mathrm{CD} 8^{+} \mathrm{T}$ cells were sorted using the MagniSort Mouse CD4 Naive T cell Enrichment Kit (8804-6824-74, Thermo Fisher Scientific) and the Naive CD8a ${ }^{+}$T Cell Isolation Kit (30-096-543, Miltenyi Biotec), respectively. The sorted cells were stimulated with anti-CD3 $(1 \mu \mathrm{g} / \mathrm{mL})$ and anti-CD28 $(1 \mu \mathrm{g} /$ $\mathrm{mL})$ antibodies in 96-well plates $\left(1 \times 10^{5}\right.$ cells per well $)$ for flow cytometric analysis, or stimulated with anti-CD3 $(1 \mu \mathrm{g} / \mathrm{mL})$ and anti-CD28 $(1 \mu \mathrm{g} / \mathrm{mL})$ antibodies in 6-well plates $\left(5 \times 10^{6}\right.$ cells per well) for immunoblot analysis or metabolic analysis.

Tumor models. MC38 and MC38-OVA murine colon cancer cells were injected s.c. into 6 - to 8 -week-old mice $\left(5 \times 10^{5}\right.$ cells per mouse $)$. B16-F10 melanoma cells were injected s.c. into 6- to 8-week-old mice $\left(5 \times 10^{5}\right.$ cells per mouse). To minimize individual variations, 6 to 8 age- and sex-matched mice in each group were used. For the adoptive transfer experiments, OT-I and OT-II cells $\left(2 \times 10^{6}\right.$ cells per mouse) were injected i.v. into MC38-OVA tumor-bearing mice on 7 day after tumor injection. The tumor-challenged mice were monitored for tumor size (tumor area indicates tumor size). Tumor-induced lethality was defined as a tumor size reaching $225 \mathrm{~mm}^{2}$.

RNA-Seq analysis. Naive CD8 ${ }^{+} \mathrm{T}$ cells isolated from the spleens of WT and KO mice were stimulated with anti-CD3 $(1 \mu \mathrm{g} / \mathrm{mL})$ and antiCD28 $(1 \mu \mathrm{g} / \mathrm{mL})$ antibodies for 8 hours in vitro. Fresh tumor-infiltrating WT and $\mathrm{KO} \mathrm{CD}^{+} \mathrm{T}$ cells were isolated from the tumor-bearing mice injected s.c. with MC38 colon cancer cells (on day 7 after injection). Total RNA was isolated from in vitro-activated $\mathrm{CD} 8^{+} \mathrm{T}$ cells and in vivo tumor-infiltrating CD8 ${ }^{+} \mathrm{T}$ cells using TRIzol (Invitrogen, Thermo Fisher Scientific) and subjected to RNA-Seq using the Illumina NextSeq 500 System. The RNA-Seq data reported here are deposited in the NCBI's Sequence Read Archive (SRA) under BioProject accession numbers PRJNA745501 and PRJNA745514.

Metabolic measurement. Seahorse XFe96 Extracellular Flux Analyzer (Agilent Technologies) was used for metabolic analysis. Naive $\mathrm{CD}^{+} \mathrm{T}$ cells stimulated with anti-CD3 $(1 \mu \mathrm{g} / \mathrm{mL})$ and anti-CD28 (1 $\mu \mathrm{g} / \mathrm{mL}$ ) antibodies for 8 hours or tumor-infiltrating $\mathrm{CD}^{+} \mathrm{T}$ cells from human CRC samples treated or not with NAC were seeded at a density of $2 \times 10^{5}$ cells per well. The extracellular acidification rate (ECAR) and oxygen consumption rate (OCR) for each well were calculated, while the cells were subjected to the XF Glycolytic stress or the XF Cell Mito test using the following concentrations of injected compounds: $10 \mathrm{mM}$ glucose, $2 \mu \mathrm{M}$ oligomycin, $50 \mathrm{mM}$ 2-deoxy-D-glucose (2-DG), $1 \mu \mathrm{M}$ carbonyl cyanide 4-(trifluoromethoxy)phenylhydrazone (FCCP), and $0.5 \mu \mathrm{M}$ rotenone/antimycin A. The XF Glycolytic stress or the XF Cell Mito test kits were purchased from Agilent Technologies.

Immunoblotting, immunoprecipitation, and ELISA. T cells were washed with ice-cold PBS and lysed on ice for 30 minutes in RIPA buffer containing protease inhibitor. Cell lysates were immunoprecipitated with the appropriate antibodies using protein A/G agarose beads. Samples were then used for immunoblot analysis with the indicated antibodies. PI3K activity was detected using the PI3-Kinase Activity ELISA Kit (K-1000s, Echelon) according to the manufacturer's instructions.

Statistics. Statistical analysis was performed using GraphPad Prism (GraphPad Software). Two-way ANOVA, where applicable, was performed to compare continuous outcomes across multiple experimental groups. One-way ANOVA and 2-tailed, unpaired Student's $t$ tests were also performed. Survival curves were analyzed by log-rank (Mantel-Cox) test. A P value of less than 0.05 was considered statistically significant.

Study approval. The patient-derived PBMC and human colorectal cancer sample collection was approved by the Clinical Research Ethics Committee of Ruijin Hospital and complied with all relevant ethics regulations. Informed consent was obtained from each patient, and the study protocol was approved by the Clinical Research Ethics Committee of Ruijin Hospital and complied with all relevant ethics regulations. All animal procedures were performed according to protocols approved by the IACUC of the Shanghai Jiao Tong University School of Medicine.

\section{Author contributions}

$\mathrm{Z}$. $\mathrm{Wu}$ performed the experiments, analyzed the data, and wrote the manuscript. HH, QH, ZH, XLT, RD, and YY helped with mouse experiments and flow cytometry and analyzed the data. XY, RZ, Z. Wang, and QZ designed the experiments, interpreted the results, wrote the manuscript, and oversaw the research project.

\section{Acknowledgments}

This study was supported by grants from the National Natural Science Foundation of China (31922025, 32170908, 81930040); the National Key Research and Development Program of China (2020YFA0803603); and the Shanghai Science and Technology Commission (20JC1410100).

Address correspondence to: Qiang Zou, Shanghai Institute of Immunology, Shanghai Jiao Tong University School of Medicine, 280 South Chongqing Road, Shanghai 200025, China. Phone: 086.021.64666150; Email: Qzou1984@sjtu.edu.cn. Or to: Zhengting Wang, Department of Gastroenterology, Ruijin Hospital, Shanghai Jiao Tong University School of Medicine, 197 Ruijin Er Road, Shanghai 200025, China. Phone: 086.021.64370045; Email: zhengtingwang@shsmu.edu.cn. Or to: Ren Zhao, Department of General Surgery, Ruijin Hospital, Shanghai Jiao Tong University School of Medicine, 197 Ruijin Er Road, Shanghai 200025, China. Phone: 086.021.64370045; Email: rjzhaoren@139.com. Or to: Xiaoyan Yu, Shanghai Institute of Immunology, Shanghai Jiao Tong University School of Medicine, 280 South Chongqing Road, Shanghai 200025, China. Phone: 086.021.64666150; Email:yuxy@shsmu.edu.cn. 
1. Ribas A, Wolchok JD. Cancer immunotherapy using checkpoint blockade. Science. 2018;359(6382):1350-1355.

2. Baharom $F$, et al. Intravenous nanoparticle vaccination generates stem-like TCF1+ neoantigen-specific CD8 ${ }^{+}$T cells. Nat Immunol. 2021;22(1):41-52.

3. Zhou J, et al. The ubiquitin ligase MDM2 sustains STAT5 stability to control T cell-mediated antitumor immunity. Nat Immunol. 2021;22(4):460-470.

4. Zhang L, Romero P. Metabolic control of CD8 ${ }^{+}$ $\mathrm{T}$ cell fate decisions and antitumor immunity. Trends Mol Med. 2018;24(1):30-48.

5. Zhao E, et al. Cancer mediates effector T cell dysfunction by targeting microRNAs and EZH2 via glycolysis restriction. Nat Immunol. 2016;17(1):95-103.

6. Karmaus PWF, et al. Metabolic heterogeneity underlies reciprocal fates of $\mathrm{T}_{\mathrm{H}} 17$ cell stemness and plasticity. Nature. 2019;565(7737):101-105.

7. Hamaidi I, et al. Sirt2 inhibition enhances metabolic fitness and effector functions of tumor-reactive T cells. Cell Metab. 2020;32(3):420-436.

8. Buck MD, et al. T cell metabolism drives immunity. JExp Med. 2015;212(9):1345-1360.

9. Geltink RIK, et al. Unraveling the complex interplay between $\mathrm{T}$ cell metabolism and function. Annu Rev Immunol. 2018;36:461-488.

10. Buck MD, et al. Metabolic instruction of immunity. Cell. 2017;169(4):570-586.

11. Rivadeneira DB, Delgoffe GM. Antitumor T-cell reconditioning: improving metabolic fitness for optimal cancer immunotherapy. Clin Cancer Res. 2018;24(11):2473-2481.

12. Li X, et al. Navigating metabolic pathways to enhance antitumour immunity and immunotherapy. Nat Rev Clin Oncol. 2019;16(7):425-441.

13. Wang F, et al. ZFP91 disturbs metabolic fitness and antitumor activity of tumor-infiltrating $\mathrm{T}$ cells. J Clin Invest. 2021;131(19):e144318.

14. Maj T, et al. Oxidative stress controls regulatory T cell apoptosis and suppressor activity and PD-L1-blockade resistance in tumor. Nat Immunol. 2017;18(12):1332-1341.

15. Hu Z, et al. SENP3 senses oxidative stress to facilitate STING-dependent dendritic cell antitumor function. Mol Cell. 2021;81(5):940-952.

16. Tse HM, et al. Disruption of innate-mediated proinflammatory cytokine and reactive oxygen species third signal leads to antigen-specific hyporesponsiveness. JImmunol. 2007;178(2):908-917.

17. Kaminski MM, et al. Mitochondrial reactive oxygen species control $\mathrm{T}$ cell activation by regulating IL-2 and IL-4 expression: mechanism of ciprofloxacin-mediated immunosuppression. J Immunol. 2010;184(9):4827-4841.

18. Sena LA, et al. Mitochondria are required for antigen-specific $\mathrm{T}$ cell activation through reactive oxygen species signaling. Immunity. 2013;38(2):225-236.

19. Chamoto K, et al. Mitochondrial activation chemicals synergize with surface receptor PD-1 blockade for T cell-dependent antitumor activity. Proc Natl Acad Sci U S A. 2017;114(5):E761-E770.

20. Scharping NE, et al. The tumor microenvironment represses $\mathrm{T}$ cell mitochondrial biogenesis to drive intratumoral $\mathrm{T}$ cell metabolic insufficiency and dysfunction. Immunity. 2016;45(2):374-388.

21. Scharping NE, et al. Mitochondrial stress induced by continuous stimulation under hypoxia rapidly drives T cell exhaustion. Nat Immunol. 2021;22(2):205-215

22. Hickey CM, et al. Function and regulation of SUMO proteases. Nat Rev Mol Cell Biol. 2012;13(12):755-766.

23. Flotho A, Melchior F. Sumoylation: a regulatory protein modification in health and disease. Annu Rev Biochem. 2013;82:357-385.

24. Feligioni M, Nistico R. SUMO: a (oxidative) stressed protein. Neuromolecular Med. 2013;15(4):707-719.

25. Huang C, et al. SENP3 is responsible for HIF-1 transactivation under mild oxidative stress via $\mathrm{p} 300$ de-SUMOylation. ЕMBO J. 2009;28(18):2748-2762.

26. Yan S, et al. Redox regulation of the stability of the SUMO protease SENP3 via interactions with CHIP and Hsp90. EMBO J. 2010;29(22):3773-3786.

27. Yu X, et al. SENP3 maintains the stability and function of regulatory $\mathrm{T}$ cells via $\mathrm{BACH} 2 \mathrm{deSU}-$ MOylation. Nat Commun. 2018;9(1):3157.

28. Liu K, et al. A fine-tuning mechanism underlying self-control for autophagy: deSUMOylation of BECN1 by SENP3. Autophagy. 2020;16(6):975-990.

29. Bawa-Khalfe T, et al. Differential expression of SUMO-specific protease 7 variants regulates epithelial-mesenchymal transition. Proc Natl Acad Sci U S A. 2012;109(43):17466-17471.

30. Garvin AJ, et al. The deSUMOylase SENP7 promotes chromatin relaxation for homologous recombination DNA repair. EMBO Rep. 2013;14(11):975-983.

31. Cui Y, et al. SENP7 potentiates cGAS activation by relieving SUMO-mediated inhibition of cytosolic DNA sensing. PLoS Pathog. 2017;13(1):e1006156.

32. Barry R, et al. SUMO-mediated regulation of NLRP3 modulates inflammasome activity. Nat Commun. 2018;9(1):3001.

33. Gu M, et al. NF-kappaB-inducing kinase maintains T cell metabolic fitness in antitumor immunity. Nat Immunol. 2021;22(2):193-204.

34. $\mathrm{Hu} \mathrm{Z}$, et al. Acylglycerol kinase maintains metabolic state and immune responses of $\mathrm{CD}^{+} \mathrm{T}$ cells. Cell Metab. 2019;30(2):290-302.e5.

35. Yang J, et al. Targeting PI3K in cancer: mechanisms and advances in clinical trials. Mol Cancer. 2019;18(1):26.

36. Swatek KN, Komander D. Ubiquitin modifications. Cell Res. 2016;26(4):399-422.

37. Pollizzi KN, Powell JD. Integrating canonical and metabolic signalling programmes in the regulation of T cell responses. Nat Rev Immunol. 2014;14(7):435-446.

38. Saxton RA, Sabatini DM. mTOR signaling in growth, metabolism, and disease. Cell. 2017;168(6):960-976.

39. Maison C, et al. The SUMO protease SENP7 is a critical component to ensure HP1 enrichment at pericentric heterochromatin. Nat Struct Mol Biol. 2012;19(4):458-460.

40. Romeo K, et al. The SENP7 SUMO-protease presents a module of two HP1 interaction motifs that locks HP1 protein at pericentric heterochromatin. Cell Rep. 2015;10(5):771-782.

41. Zhu H, et al. SPOP E3 ubiquitin ligase adaptor promotes cellular senescence by degrading the SENP7 deSUMOylase. Cell Rep. 2015;13(6):1183-1193.

42. Suhail A, et al. DeSUMOylase SENP7-mediated epithelial signaling triggers intestinal inflammation via expansion of gamma-delta T cells. Cell Rep. 2019;29(11):3522-3538.

43. MacIver NJ, et al. Metabolic regulation of T lymphocytes. Annu Rev Immunol. 2013;31:259-283.

44. Taniuchi I. CD4 Helper and CD8 cytotoxic T cell differentiation. Annu Rev Immunol. 2018;36:579-601.

45. Yang $\mathrm{W}$, et al. Potentiating the antitumour response of CD8(+) T cells by modulating cholesterol metabolism. Nature. 2016;531(7596):651-655. 